Supplement of Adv. Stat. Clim. Meteorol. Oceanogr., 1, 29-44, 2015

http://www.adv-stat-clim-meteorol-oceanogr.net/1/29/2015/

doi:10.5194/ascmo-1-29-2015-supplement

(C) Author(s) 2015. CC Attribution 3.0 License.

(c) (i)

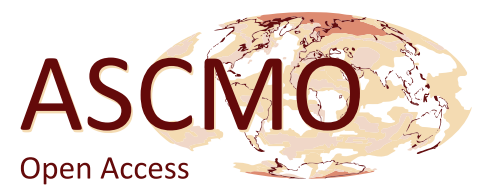

Supplement of

\title{
Bivariate spatial analysis of temperature and precipitation from general circulation models and observation proxies
}

\author{
R. Philbin and M. Jun \\ Correspondence to: M. Jun (mjun@stat.tamu.edu)
}

The copyright of individual parts of the supplement might differ from the CC-BY 3.0 licence. 

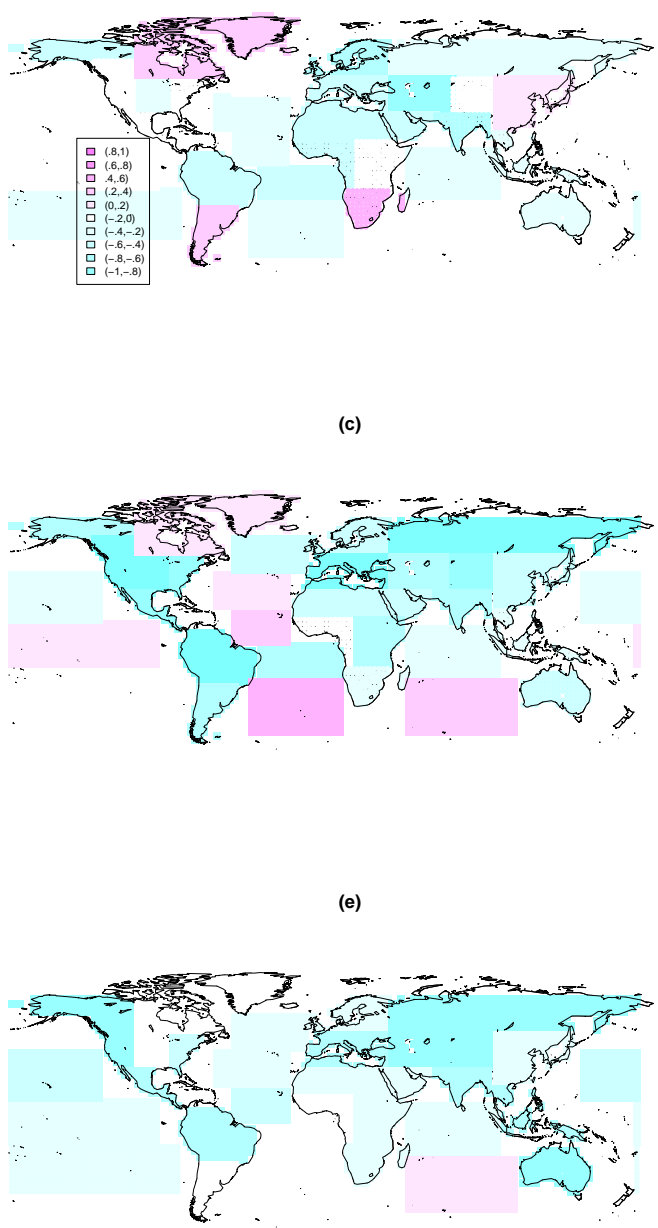

(b)

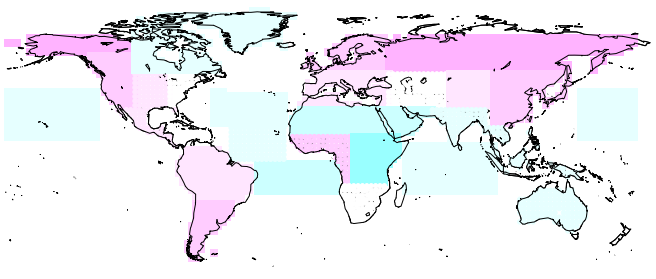

(d)

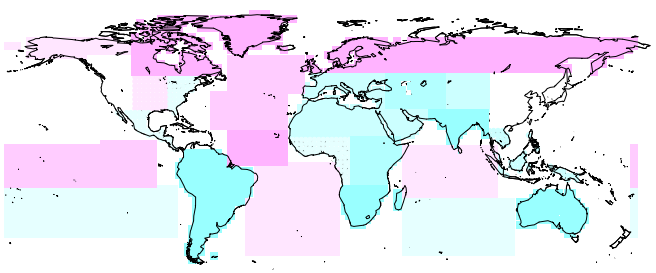

(f)

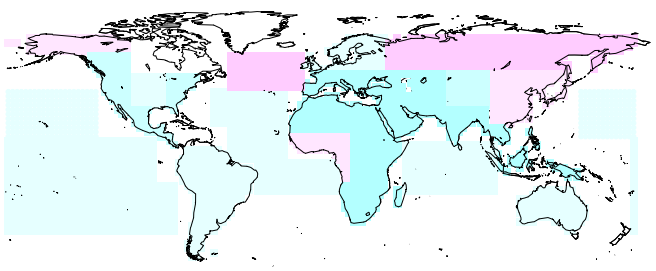

Figure 1: Correlation coefficient maps (a) JJA and (b) DJF for NCEP/GPCP, (c) JJA and (d) DJF for GFDL, and (e) JJA and (f) DJF for NCAR. 

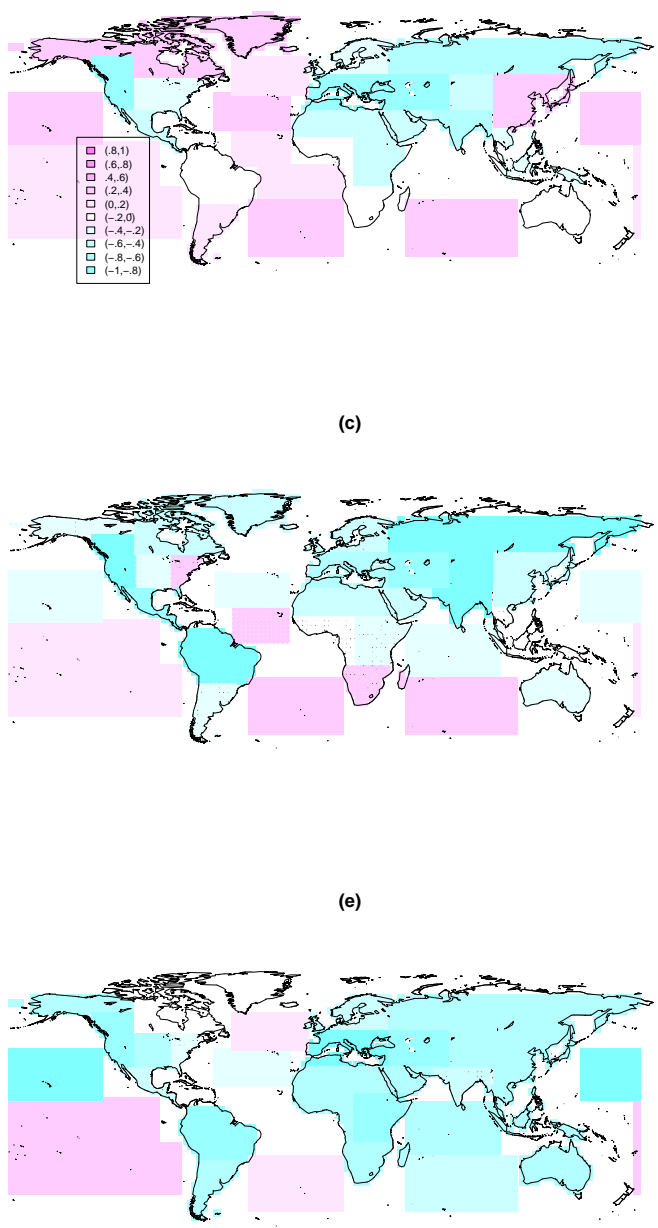

(b)

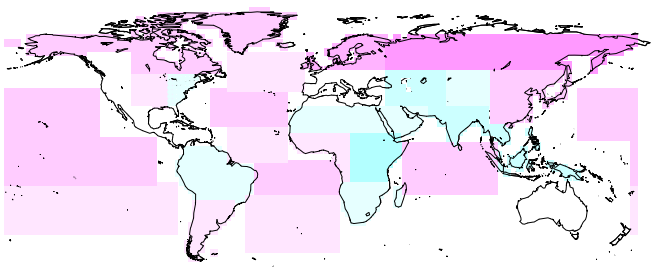

(d)

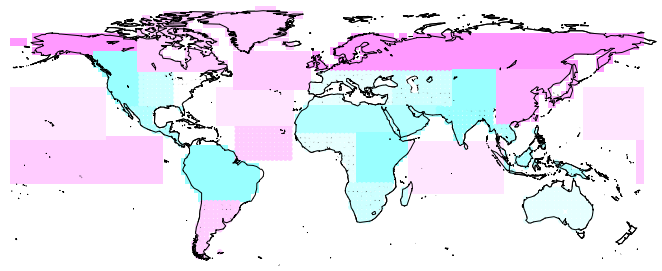

(f)

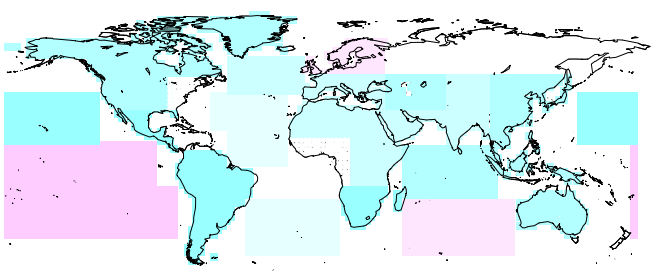

Figure 2: Correlation coefficient maps: (a) JJA and (b) DJF for GEMS, (c) JJA and (d) DJF for BCC, and (e) JJA and (f) DJF for GEOS. 

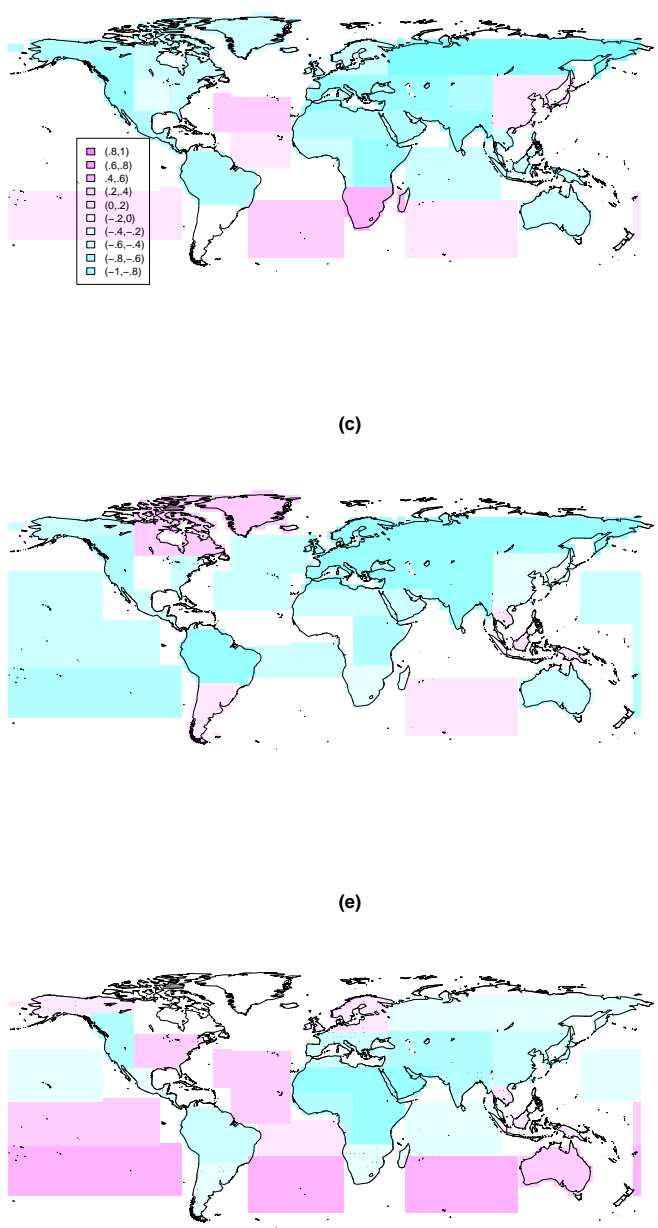

(b)

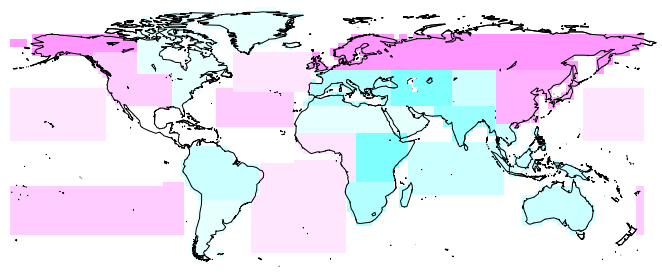

(d)

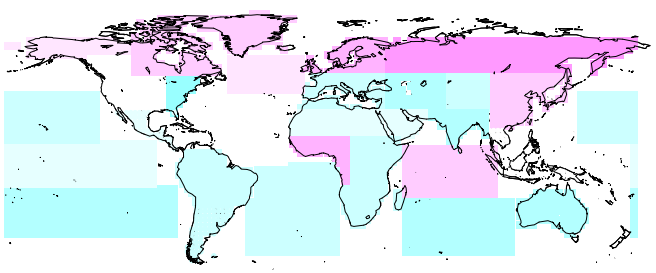

(f)

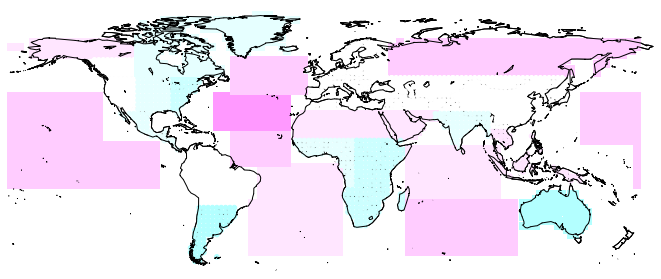

Figure 3: Correlation coefficient maps: (a) JJA and (b) DJF for MIROC, (c) JJA and (d) DJF for MPI, and (e) JJA and (f) DJF for HAD. 
Table 1: Parameter estimates for Alaska (ALA) by season with asymptotic standard errors in parentheses

\begin{tabular}{rrrrrrrr}
\hline & $\sigma_{P}$ & $\sigma_{T}$ & $\nu_{P}$ & $\nu_{T}$ & $a^{-1}$ & $\rho$ \\
\hline NCEP/GPCP & $2.4(0.7)$ & $1.9(0.4)$ & $0.85(0.14)$ & $0.71(0.14)$ & $628(292)$ & $-0.362(0.093)$ \\
BCC & $1.9(0.6)$ & $2.4(0.6)$ & $0.72(0.13)$ & $0.82(0.14)$ & $883(467)$ & $-0.155(0.12)$ \\
GEMS & $3.2(0.5)$ & $2.8(0.4)$ & $1.4(0.13)$ & $1.31(0.14)$ & $217(43)$ & $0.544(0.042)$ \\
GEOS & $4.8(1.3)$ & $5.1(1.7)$ & $0.38(0.06)$ & $0.61(0.05)$ & $1580(1090)$ & $-0.512(0.073)$ \\
GFDL & $3.8(1.1)$ & $3.4(1.1)$ & $0.74(0.1)$ & $0.82(0.13)$ & $760(375)$ & $-0.563(0.076)$ \\
HAD & $2.8(0.5)$ & $3.3(0.6)$ & $0.67(0.2)$ & $0.86(0.18)$ & $445(208)$ & $0.293(0.115)$ \\
MIROC & $4.7(0.8)$ & $6.2(1.7)$ & $0.76(0.07)$ & $1.27(0.04)$ & $479(128)$ & $-0.478(0.045)$ \\
MPI & $5(1.4)$ & $3.2(0.5)$ & $1.22(0.09)$ & $0.7(0.15)$ & $479(148)$ & $-0.278(0.065)$ \\
NCAR & $5.4(1.2)$ & $3.3(0.5)$ & $1.2(0.08)$ & $0.99(0.09)$ & $318(79)$ & $-0.617(0.029)$ \\
\hline
\end{tabular}

JJA ALA ( North )

\begin{tabular}{rrrrrrrr}
\hline & $\sigma_{P}$ & $\sigma_{T}$ & $\nu_{P}$ & $\nu_{T}$ & $a^{-1}$ & $\rho$ \\
\hline NCEP/GPCP & $6.3(1.8)$ & $3.5(0.9)$ & $0.9(0.13)$ & $0.82(0.13)$ & $751(346)$ & $0.628(0.062)$ \\
BCC & $6.6(2.3)$ & $8.3(2.8)$ & $1.37(0.15)$ & $1.48(0.13)$ & $633(200)$ & $0.949(0.016)$ \\
GEMS & $8.5(2)$ & $5.2(0.9)$ & $1.95(0.11)$ & $1.3(0.12)$ & $345(70)$ & $0.487(0.046)$ \\
GEOS & $5.5(1)$ & $18.7(8.1)$ & $0.47(0.08)$ & $1.23(0.05)$ & $906(391)$ & $-0.423(0.079)$ \\
GFDL & $7.2(3.1)$ & $2.5(0.6)$ & $1.32(0.12)$ & $0.83(0.17)$ & $749(300)$ & $0.29(0.085)$ \\
HAD & $4.4(0.9)$ & $4.9(0.5)$ & $1.02(0.33)$ & $0.24(\mathrm{NA})$ & $482(324)$ & $0.386(0.092)$ \\
MIROC & $3.8(0.6)$ & $5.4(1)$ & $1.29(0.07)$ & $1.83(0.05)$ & $292(43)$ & $0.834(0.018)$ \\
MPI & $7.3(2.2)$ & $2.7(0.5)$ & $1.72(0.09)$ & $1.18(0.12)$ & $509(120)$ & $0.386(0.061)$ \\
NCAR & $4.4(0.6)$ & $4.5(0.7)$ & $1.24(0.07)$ & $1.5(0.07)$ & $235(39)$ & $0.321(0.041)$ \\
\hline
\end{tabular}

DJF ALA ( North ) 
Table 2: Parameter estimates for Western North America (WNA) by season with asymptotic standard errors in parentheses

\begin{tabular}{rrrrrrrr}
\hline & $\sigma_{P}$ & $\sigma_{T}$ & $\nu_{P}$ & $\nu_{T}$ & $a^{-1}$ & $\rho$ \\
\hline NCEP/GPCP & $6.6(2.3)$ & $4.4(1.6)$ & $0.93(0.15)$ & $0.99(0.16)$ & $944(469)$ & $0.031(0.105)$ \\
BCC & $13.7(5.1)$ & $6.3(2.4)$ & $1.22(0.15)$ & $1.11(0.18)$ & $1124(478)$ & $-0.884(0.031)$ \\
GEMS & $6.3(1.3)$ & $2.9(0.5)$ & $1.67(0.16)$ & $1.36(0.16)$ & $288(68)$ & $-0.735(0.029)$ \\
GEOS & $14.9(6.3)$ & $10.1(4.5)$ & $0.72(0.08)$ & $0.7(0.11)$ & $2287(1522)$ & $-0.717(0.047)$ \\
GFDL & $10.5(3)$ & $6(1.8)$ & $1.16(0.18)$ & $1.09(0.21)$ & $625(262)$ & $-0.851(0.03)$ \\
HAD & $6.2(2)$ & $3.3(1)$ & $0.71(0.15)$ & $0.63(0.19)$ & $1173(784)$ & $-0.461(0.095)$ \\
MIROC & $18.5(7.6)$ & $16.3(7)$ & $1(0.07)$ & $1.05(0.06)$ & $1545(713)$ & $-0.786(0.023)$ \\
MPI & $9.7(2.9)$ & $2.4(0.3)$ & $1.57(0.19)$ & $0.5(0.3)$ & $466(157)$ & $-0.417(0.06)$ \\
NCAR & $16.5(6.2)$ & $10.5(3.5)$ & $1.11(0.07)$ & $1.02(0.07)$ & $913(363)$ & $-0.69(0.024)$ \\
\hline
\end{tabular}

JJA WNA ( Mid-N )

\begin{tabular}{rrrrrrrr}
\hline & $\sigma_{P}$ & $\sigma_{T}$ & $\nu_{P}$ & $\nu_{T}$ & $a^{-1}$ & $\rho$ \\
\hline NCEP/GPCP & $13.7(5.9)$ & $5.5(2.3)$ & $0.57(0.09)$ & $0.58(0.09)$ & $2904(2417)$ & $0.416(0.086)$ \\
BCC & $30(16.8)$ & $8.5(2.5)$ & $1.98(0.14)$ & $0.95(0.2)$ & $1595(595)$ & $-0.69(0.061)$ \\
GEMS & $11.8(3.3)$ & $2.7(0.4)$ & $1.97(0.16)$ & $0.86(0.19)$ & $457(115)$ & $0.178(0.059)$ \\
GEOS & $14.7(5.6)$ & $18.5(11)$ & $0.48(0.07)$ & $0.78(0.06)$ & $4480(3440)$ & $-0.405(0.079)$ \\
GFDL & $8.8(2.2)$ & $2.5(0.3)$ & $2.15(0.35)$ & $0.74(0.4)$ & $329(100)$ & $0.148(0.09)$ \\
HAD & $13.4(6)$ & $5.6(2.1)$ & $0.66(0.12)$ & $0.56(0.13)$ & $2598(1961)$ & $0.109(0.117)$ \\
MIROC & $6.9(1.2)$ & $3.6(0.4)$ & $2.06(0.11)$ & $1(0.15)$ & $335(51)$ & $0.54(0.041)$ \\
MPI & $9.6(2.1)$ & $2.4(0.2)$ & $2.21(0.2)$ & $0.83(0.26)$ & $284(56)$ & $0.084(0.075)$ \\
NCAR & $11.8(2.7)$ & $3.7(0.4)$ & $1.47(0.1)$ & $0.89(0.15)$ & $330(77)$ & $-0.282(0.04)$ \\
\hline
\end{tabular}

\section{DJF WNA ( Mid-N )}


Table 3: Parameter estimates for Central North America (CNA) by season with asymptotic standard errors in parentheses

\begin{tabular}{rrrrrrrr}
\hline & $\sigma_{P}$ & $\sigma_{T}$ & $\nu_{P}$ & $\nu_{T}$ & $a^{-1}$ & $\rho$ \\
\hline NCEP/GPCP & $2.2(0.3)$ & $2.6(0.6)$ & $2.12(0.29)$ & $5(49.23)$ & $203(17)$ & $-0.002(0.131)$ \\
BCC & $3.4(1.1)$ & $1.8(0.4)$ & $3.21(0.99)$ & $1.95(0.52)$ & $276(106)$ & $-0.169(0.147)$ \\
GEMS & $3.7(1)$ & $2.2(0.5)$ & $1.37(0.14)$ & $1.44(0.14)$ & $459(128)$ & $-0.017(0.076)$ \\
GEOS & $4.7(1)$ & $1.8(0.4)$ & $1.16(0.2)$ & $0.88(0.26)$ & $453(167)$ & $-0.579(0.068)$ \\
GFDL & $10(4.1)$ & $3.2(1.2)$ & $1.55(0.18)$ & $1.13(0.21)$ & $893(378)$ & $-0.799(0.043)$ \\
HAD & $3.1(0.6)$ & $2.8(0.8)$ & $0.64(0.25)$ & $1.07(0.23)$ & $474(268)$ & $0.538(0.099)$ \\
MIROC & $3.8(0.9)$ & $2(0.4)$ & $1.58(0.14)$ & $1.24(0.15)$ & $359(81)$ & $-0.043(0.07)$ \\
MPI & $3.6(0.7)$ & $1.5(0.3)$ & $2.05(0.33)$ & $2.33(0.33)$ & $200(42)$ & $0.009(0.094)$ \\
NCAR & $4.2(1)$ & $2.7(0.6)$ & $1.31(0.09)$ & $1.11(0.09)$ & $542(136)$ & $0.063(0.053)$ \\
\hline
\end{tabular}

JJA CNA ( Mid-N )

\begin{tabular}{rrrrrrrr}
\hline \multicolumn{1}{c}{$\sigma_{P}$} & $\sigma_{T}$ & $\nu_{P}$ & $\nu_{T}$ & $a^{-1}$ & $\rho$ \\
\hline NCEP/GPCP & $3.2(0.5)$ & $2.6(0.8)$ & $2.11(0.53)$ & $4.67(3.79)$ & $174(43)$ & $0.333(0.133)$ \\
BCC & $3.2(1.1)$ & $3.5(1.4)$ & $1.5(0.24)$ & $2.07(0.24)$ & $697(246)$ & $-0.171(0.163)$ \\
GEMS & $2.7(0.5)$ & $2(0.5)$ & $1.15(0.15)$ & $1.26(0.15)$ & $404(125)$ & $0.247(0.071)$ \\
GEOS & $3.9(0.8)$ & $3.8(1.1)$ & $0.83(0.15)$ & $1.44(0.14)$ & $535(191)$ & $-0.526(0.073)$ \\
GFDL & $3.7(1.4)$ & $1.8(0.6)$ & $1.16(0.19)$ & $1.17(0.2)$ & $723(332)$ & $0.24(0.112)$ \\
HAD & $3.2(0.6)$ & $2.4(0.5)$ & $0.83(0.29)$ & $0.46(0.63)$ & $425(237)$ & $-0.132(0.133)$ \\
MIROC & $4.4(1.2)$ & $5.2(2)$ & $1.2(0.12)$ & $1.56(0.1)$ & $501(162)$ & $0.577(0.049)$ \\
MPI & $3.2(0.6)$ & $2.8(0.7)$ & $1.59(0.22)$ & $2.5(0.22)$ & $299(63)$ & $0.098(0.103)$ \\
NCAR & $3.1(0.7)$ & $2.3(0.5)$ & $1.16(0.1)$ & $1.24(0.09)$ & $438(117)$ & $-0.048(0.053)$ \\
\hline
\end{tabular}

DJF CNA ( Mid-N ) 
Table 4: Parameter estimates for Eastern North America (ENA) by season with asymptotic standard errors in parentheses

\begin{tabular}{rrrrrrrr}
\hline & $\sigma_{P}$ & $\sigma_{T}$ & $\nu_{P}$ & $\nu_{T}$ & $a^{-1}$ & $\rho$ \\
\hline NCEP/GPCP & $1.8(0.5)$ & $1.1(0.4)$ & $1.39(0.72)$ & $2.31(0.75)$ & $277(161)$ & $0.193(0.175)$ \\
BCC & $3.4(1.6)$ & $3.2(1.4)$ & $1.26(0.29)$ & $1(0.35)$ & $995(709)$ & $0.559(0.123)$ \\
GEMS & $4.2(1.8)$ & $1(0.3)$ & $0.91(0.16)$ & $0.7(0.15)$ & $838(510)$ & $-0.173(0.1)$ \\
GEOS & $6.7(2.3)$ & $1(0.2)$ & $1.21(0.25)$ & $0.42(0.41)$ & $596(319)$ & $-0.135(0.127)$ \\
GFDL & $4.3(1.5)$ & $1.9(0.5)$ & $1.7(0.49)$ & $1.17(0.51)$ & $349(190)$ & $-0.785(0.068)$ \\
HAD & $1.6(7.6)$ & $1.2(7.6)$ & $4.71(\mathrm{NA})$ & $5(0)$ & $3(0)$ & $0.43(2.78)$ \\
MIROC & $3.6(0.8)$ & $1.1(0.2)$ & $1.83(0.36)$ & $1.02(0.36)$ & $176(58)$ & $-0.29(0.087)$ \\
MPI & $1.5(0.4)$ & $1(0.2)$ & $1.05(0.36)$ & $0.93(0.33)$ & $294(149)$ & $-0.296(0.112)$ \\
NCAR & $1.7(0.4)$ & $1(0.2)$ & $0.97(0.14)$ & $0.59(0.17)$ & $336(134)$ & $-0.267(0.066)$ \\
\hline
\end{tabular}

JJA ENA ( Mid-N )

\begin{tabular}{rrrrrrr}
\hline & $\sigma_{P}$ & $\sigma_{T}$ & $\nu_{P}$ & $\nu_{T}$ & $a^{-1}$ & $\rho$ \\
\hline NCEP/GPCP & $3.6(1.2)$ & $1.5(0.4)$ & $1.13(0.29)$ & $0.5(0.67)$ & $501(296)$ & $0.196(0.155)$ \\
BCC & $5.5(3.3)$ & $1.6(0.2)$ & $1.84(0.46)$ & $0.1(\mathrm{NA})$ & $838(520)$ & $0.119(0.241)$ \\
GEMS & $2.7(0.7)$ & $1.2(0.2)$ & $2.47(0.57)$ & $0.95(0.49)$ & $194(70)$ & $-0.134(0.104)$ \\
GEOS & $3.6(1.5)$ & $3.3(1.6)$ & $0.81(0.17)$ & $0.81(0.29)$ & $900(630)$ & $0.069(0.14)$ \\
GFDL & $3.7(1.7)$ & $1.4(0.4)$ & $1.31(0.39)$ & $0.37(0.81)$ & $624(485)$ & $-0.171(0.159)$ \\
HAD & $2.4(0.4)$ & $2.4(0.3)$ & $0.75(0.63)$ & $0.1(33.57)$ & $242(206)$ & $-0.305(0.175)$ \\
MIROC & $3.2(0.9)$ & $2.7(0.8)$ & $1.09(0.2)$ & $0.9(0.18)$ & $429(203)$ & $-0.178(0.093)$ \\
MPI & $4(1.1)$ & $1.1(0.4)$ & $1.13(0.29)$ & $0.67(0.44)$ & $457(251)$ & $-0.699(0.075)$ \\
NCAR & $3.4(1.3)$ & $8.5(4.5)$ & $0.75(0.09)$ & $0.95(0.1)$ & $1399(810)$ & $-0.348(0.07)$ \\
\hline
\end{tabular}

DJF ENA ( Mid-N )

Table 5: Parameter estimates for Greenland (GRL) by season with asymptotic standard errors in parentheses

\begin{tabular}{rrrrrrrr}
\hline & $\sigma_{P}$ & $\sigma_{T}$ & $\nu_{P}$ & $\nu_{T}$ & $a^{-1}$ & $\rho$ \\
\hline NCEP/GPCP & $3.9(1.5)$ & $2.4(0.6)$ & $0.55(0.05)$ & $0.34(0.09)$ & $2318(1760)$ & $0.402(0.061)$ \\
BCC & $1.9(0.3)$ & $3.5(0.5)$ & $1.18(0.1)$ & $0.7(0.16)$ & $376(89)$ & $-0.323(0.072)$ \\
GEMS & $4.4(1.2)$ & $5(1.1)$ & $0.67(0.04)$ & $0.52(0.04)$ & $1187(536)$ & $0.566(0.03)$ \\
GEOS & $4(0.3)$ & $4.6(0.7)$ & $0.17(0.04)$ & $0.43(0.01)$ & $663(293)$ & $0.045(0.061)$ \\
GFDL & $3.9(0.6)$ & $2.9(0.2)$ & $1.86(0.13)$ & $0.63(0.2)$ & $209(34)$ & $0.282(0.064)$ \\
HAD & $3.2(0.4)$ & $2.2(0.2)$ & $0.7(0.14)$ & $0.44(0.18)$ & $353(130)$ & $0.135(0.083)$ \\
MIROC & $3.7(0.7)$ & $4.1(0.5)$ & $1.29(0.04)$ & $0.68(0.06)$ & $550(102)$ & $-0.203(0.039)$ \\
MPI & $3.9(0.6)$ & $4.5(0.5)$ & $1.05(0.04)$ & $0.62(0.08)$ & $419(80)$ & $0.511(0.042)$ \\
NCAR & $4.4(0.5)$ & $2(0.1)$ & $1.48(0.04)$ & $0.72(0.06)$ & $188(19)$ & $0.079(0.03)$ \\
\hline
\end{tabular}

JJA GRL ( North )

\begin{tabular}{rrrrrrr}
\hline \multicolumn{1}{c}{$\sigma_{P}$} & $\sigma_{T}$ & $\nu_{P}$ & $\nu_{T}$ & $a^{-1}$ & $\rho$ \\
\hline NCEP/GPCP & $7.9(1.8)$ & $28.1(12.8)$ & $0.82(0.06)$ & $1.59(0.04)$ & $1096(356)$ & $-0.114(0.079)$ \\
BCC & $3.4(1)$ & $3.5(0.6)$ & $1.57(0.06)$ & $0.78(0.12)$ & $766(180)$ & $0.493(0.062)$ \\
GEMS & $10.6(3.9)$ & $8.9(2.1)$ & $0.98(0.03)$ & $0.65(0.05)$ & $1494(586)$ & $0.527(0.031)$ \\
GEOS & $8(1.6)$ & $52.8(25.8)$ & $0.5(0.05)$ & $1.19(0.03)$ & $1663(766)$ & $-0.479(0.055)$ \\
GFDL & $7.1(1.5)$ & $5(0.7)$ & $1.57(0.07)$ & $1.12(0.09)$ & $434(82)$ & $0.61(0.043)$ \\
HAD & $9.1(3.6)$ & $9.5(2.1)$ & $0.84(0.09)$ & $0.39(0.21)$ & $1439(864)$ & $-0.182(0.09)$ \\
MIROC & $12.9(4)$ & $30.8(11)$ & $1.17(0.03)$ & $1.33(0.02)$ & $1709(468)$ & $-0.125(0.043)$ \\
MPI & $11.3(3.4)$ & $8.7(1.7)$ & $1.21(0.04)$ & $0.9(0.05)$ & $894(242)$ & $0.597(0.035)$ \\
NCAR & $10.1(1.7)$ & $7.8(0.9)$ & $1.61(0.04)$ & $1.36(0.04)$ & $298(36)$ & $0.036(0.032)$ \\
\hline
\end{tabular}

DJF GRL ( North ) 
Table 6: Parameter estimates for Northern Europe (NEU) by season with asymptotic standard errors in parentheses

\begin{tabular}{rrrrrrr}
\hline & $\sigma_{P}$ & $\sigma_{T}$ & $\nu_{P}$ & $\nu_{T}$ & $a^{-1}$ & $\rho$ \\
\hline NCEP/GPCP & $3.3(1.6)$ & $1.9(0.7)$ & $0.44(0.11)$ & $0.43(0.08)$ & $3619(3723)$ & $-0.484(0.078)$ \\
BCC & $3.5(1.8)$ & $2.6(0.7)$ & $0.98(0.14)$ & $0.61(0.22)$ & $1379(912)$ & $-0.355(0.113)$ \\
GEMS & $3.7(0.9)$ & $1.3(0.2)$ & $1.08(0.14)$ & $0.88(0.14)$ & $417(136)$ & $-0.182(0.06)$ \\
GEOS & $4.7(1.3)$ & $3.6(1.2)$ & $0.67(0.09)$ & $0.9(0.1)$ & $901(440)$ & $-0.38(0.075)$ \\
GFDL & $3(0.8)$ & $1.5(0.3)$ & $0.5(0.1)$ & $0.58(0.12)$ & $831(548)$ & $-0.435(0.078)$ \\
HAD & $2.3(0.4)$ & $2.1(0.3)$ & $0.76(0.31)$ & $0.5(0.46)$ & $359(217)$ & $0.371(0.11)$ \\
MIROC & $5.9(2.2)$ & $3.5(1.2)$ & $0.62(0.07)$ & $0.72(0.06)$ & $1475(919)$ & $-0.319(0.052)$ \\
MPI & $11.2(7.4)$ & $1.8(0.5)$ & $0.8(0.09)$ & $0.38(0.13)$ & $2523(2209)$ & $-0.668(0.042)$ \\
NCAR & $8.4(4.7)$ & $3.3(1.3)$ & $0.66(0.06)$ & $0.53(0.07)$ & $3038(2560)$ & $-0.194(0.042)$ \\
\hline
\end{tabular}

\section{JJA NEU ( North )}

\begin{tabular}{rrrrrrr}
\hline & $\sigma_{P}$ & $\sigma_{T}$ & $\nu_{P}$ & $\nu_{T}$ & $a^{-1}$ & $\rho$ \\
\hline NCEP/GPCP & $8.2(1.7)$ & $9(2.6)$ & $1.26(0.19)$ & $2.55(0.15)$ & $409(85)$ & $0.442(0.087)$ \\
BCC & $4.6(1.3)$ & $7.9(2.4)$ & $1.57(0.22)$ & $1.63(0.23)$ & $556(185)$ & $0.917(0.026)$ \\
GEMS & $25.7(13.9)$ & $7.3(2.5)$ & $1.3(0.1)$ & $0.87(0.09)$ & $1345(618)$ & $0.61(0.04)$ \\
GEOS & $4.8(1.2)$ & $11.1(5.4)$ & $0.21(0.06)$ & $0.5(0.02)$ & $5258(5240)$ & $0.284(0.087)$ \\
GFDL & $3.2(0.6)$ & $4.6(1.1)$ & $0.86(0.15)$ & $1.44(0.14)$ & $436(142)$ & $0.687(0.05)$ \\
HAD & $2.8(1)$ & $5.5(2)$ & $0.29(0.09)$ & $0.32(0.07)$ & $4252(4622)$ & $0.147(0.126)$ \\
MIROC & $8(1.7)$ & $9(1.8)$ & $1.51(0.09)$ & $1.75(0.07)$ & $413(74)$ & $0.811(0.02)$ \\
MPI & $5.6(1.4)$ & $5.5(1.2)$ & $1.08(0.12)$ & $1.35(0.1)$ & $474(137)$ & $0.676(0.047)$ \\
NCAR & $11.1(3.6)$ & $20.2(8.6)$ & $1.02(0.06)$ & $1.38(0.05)$ & $955(337)$ & $-0.133(0.045)$ \\
\hline
\end{tabular}

\section{DJF NEU ( North )}


Table 7: Parameter estimates for Northern Asia (NAS) by season with asymptotic standard errors in parentheses

\begin{tabular}{rrrrrrrr}
\hline & $\sigma_{P}$ & $\sigma_{T}$ & $\nu_{P}$ & $\nu_{T}$ & $a^{-1}$ & $\rho$ \\
\hline NCEP/GPCP & $8.2(4.2)$ & $3.2(1)$ & $0.78(0.05)$ & $0.48(0.07)$ & $4298(2925)$ & $-0.161(0.05)$ \\
BCC & $7(2.2)$ & $11.1(4.2)$ & $0.78(0.06)$ & $0.92(0.06)$ & $2120(1021)$ & $-0.84(0.019)$ \\
GEMS & $6.5(1.5)$ & $5.7(1)$ & $1.2(0.05)$ & $0.91(0.07)$ & $727(169)$ & $-0.55(0.023)$ \\
GEOS & $14.4(5.6)$ & $13.7(6.6)$ & $0.61(0.03)$ & $0.75(0.04)$ & $6272(4093)$ & $-0.601(0.03)$ \\
GFDL & $17.3(7.6)$ & $13.5(6.2)$ & $0.78(0.05)$ & $0.84(0.04)$ & $4819(2711)$ & $-0.791(0.019)$ \\
HAD & $4.5(1.3)$ & $5.9(2)$ & $0.45(0.06)$ & $0.59(0.05)$ & $3284(2187)$ & $-0.115(0.059)$ \\
MIROC & $13.7(4.7)$ & $14.7(5.5)$ & $0.84(0.03)$ & $0.94(0.03)$ & $2367(996)$ & $-0.822(0.01)$ \\
MPI & $14.7(5.3)$ & $5.3(1.2)$ & $1.2(0.05)$ & $0.84(0.07)$ & $1065(360)$ & $-0.795(0.015)$ \\
NCAR & $11.9(5.1)$ & $11.2(4.4)$ & $0.73(0.02)$ & $0.7(0.03)$ & $4122(2452)$ & $-0.727(0.011)$ \\
\hline
\end{tabular}

JJA NAS ( North )

\begin{tabular}{rrrrrrrr}
\hline & $\sigma_{P}$ & $\sigma_{T}$ & $\nu_{P}$ & $\nu_{T}$ & $a^{-1}$ & $\rho$ \\
\hline NCEP/GPCP & $6.6(1.4)$ & $5.2(0.8)$ & $0.7(0.06)$ & $0.66(0.06)$ & $1015(363)$ & $0.791(0.02)$ \\
BCC & $12.6(2.8)$ & $16.6(3.6)$ & $1.63(0.08)$ & $1.56(0.09)$ & $637(116)$ & $0.988(0.002)$ \\
GEMS & $4.4(0.4)$ & $7.6(0.8)$ & $1.44(0.05)$ & $1.22(0.05)$ & $380(41)$ & $0.828(0.011)$ \\
GEOS & $4.3(0.7)$ & $15.4(6.9)$ & $0.27(0.04)$ & $0.86(0.01)$ & $2418(1423)$ & $0.17(0.042)$ \\
GFDL & $4.7(0.7)$ & $4.7(0.5)$ & $0.98(0.07)$ & $0.81(0.08)$ & $510(110)$ & $0.796(0.018)$ \\
HAD & $3.6(0.5)$ & $4.9(0.8)$ & $0.37(0.07)$ & $0.43(0.07)$ & $1313(768)$ & $0.448(0.048)$ \\
MIROC & $14.7(2)$ & $18.4(2.5)$ & $1.71(0.03)$ & $1.7(0.03)$ & $436(42)$ & $0.977(0.002)$ \\
MPI & $6.5(0.6)$ & $6.4(0.5)$ & $1.76(0.06)$ & $1.39(0.08)$ & $249(22)$ & $0.865(0.011)$ \\
NCAR & $4.6(0.4)$ & $3.8(0.2)$ & $1.32(0.04)$ & $0.92(0.05)$ & $251(22)$ & $0.534(0.016)$ \\
\hline
\end{tabular}

DJF NAS ( North ) 
Table 8: Parameter estimates for the Mediterranean (MED) by season with asymptotic standard errors in parentheses

\begin{tabular}{rrrrrrrr}
\hline & $\sigma_{P}$ & $\sigma_{T}$ & $\nu_{P}$ & $\nu_{T}$ & $a^{-1}$ & $\rho$ \\
\hline NCEP/GPCP & $3(0.6)$ & $2.5(0.6)$ & $0.83(0.25)$ & $1.1(0.2)$ & $515(256)$ & $-0.259(0.096)$ \\
BCC & $3.1(0.6)$ & $3(0.7)$ & $1.45(0.28)$ & $1.46(0.32)$ & $466(155)$ & $-0.518(0.087)$ \\
GEMS & $3.8(0.3)$ & $2(0.2)$ & $0.96(0.17)$ & $1.11(0.16)$ & $238(56)$ & $-0.668(0.035)$ \\
GEOS & $5.3(0.6)$ & $3.6(0.4)$ & $1.2(0.28)$ & $1.35(0.29)$ & $247(70)$ & $-0.825(0.027)$ \\
GFDL & $5.5(0.9)$ & $2.8(0.4)$ & $1.29(0.27)$ & $1.09(0.31)$ & $389(134)$ & $-0.828(0.031)$ \\
HAD & $3.7(0.4)$ & $3(0.5)$ & $0.81(0.41)$ & $1.26(0.41)$ & $303(151)$ & $-0.276(0.111)$ \\
MIROC & $7.1(0.9)$ & $5.1(0.6)$ & $1.46(0.14)$ & $1.58(0.14)$ & $250(41)$ & $-0.79(0.025)$ \\
MPI & $3.7(0.4)$ & $2.5(0.2)$ & $1.33(0.27)$ & $1.38(0.26)$ & $214(52)$ & $-0.638(0.044)$ \\
NCAR & $2.9(0.3)$ & $2.8(0.2)$ & $1.02(0.13)$ & $1.05(0.13)$ & $213(43)$ & $-0.498(0.034)$ \\
\hline
\end{tabular}

JJA MED ( Mid-N )

\begin{tabular}{rrrrrrrr}
\hline & $\sigma_{P}$ & $\sigma_{T}$ & $\nu_{P}$ & $\nu_{T}$ & $a^{-1}$ & $\rho$ \\
\hline NCEP/GPCP & $5.4(1.1)$ & $2.9(0.5)$ & $0.84(0.21)$ & $0.88(0.21)$ & $549(244)$ & $0.303(0.094)$ \\
BCC & $5.5(2)$ & $4.2(1.4)$ & $1.04(0.18)$ & $0.89(0.2)$ & $999(523)$ & $-0.127(0.12)$ \\
GEMS & $7.2(1.2)$ & $2.3(0.2)$ & $1.27(0.18)$ & $0.77(0.19)$ & $357(92)$ & $0.035(0.063)$ \\
GEOS & $6.1(1.2)$ & $5.6(1.4)$ & $0.53(0.11)$ & $0.83(0.11)$ & $839(416)$ & $-0.216(0.086)$ \\
GFDL & $6.6(1.3)$ & $2.5(0.4)$ & $1.8(0.38)$ & $1.22(0.41)$ & $306(92)$ & $-0.255(0.097)$ \\
HAD & $5(0.9)$ & $3.8(0.6)$ & $0.64(0.26)$ & $0.5(0.37)$ & $555(355)$ & $0.102(0.119)$ \\
MIROC & $10.5(1.8)$ & $5.2(0.8)$ & $1.52(0.14)$ & $1.5(0.12)$ & $364(62)$ & $-0.507(0.054)$ \\
MPI & $7.3(1.2)$ & $2.8(0.4)$ & $1.15(0.22)$ & $0.91(0.21)$ & $334(97)$ & $-0.239(0.084)$ \\
NCAR & $6.5(0.7)$ & $4.1(0.4)$ & $1.28(0.11)$ & $1.24(0.11)$ & $246(38)$ & $-0.564(0.033)$ \\
\hline
\end{tabular}

DJF MED ( Mid-N ) 
Table 9: Parameter estimates for the Caspian (CAS) by season with asymptotic standard errors in parentheses

\begin{tabular}{rrrrrrr}
\hline \multicolumn{1}{c}{$\mathrm{t}$} \\
\hline NCEP/GPCP & $7.1(1)$ & $2.8(0.2)$ & $2.55(0.78)$ & $0.79(0.72)$ & $190(62)$ & $-0.604(0.056)$ \\
BCC & $3.5(0.5)$ & $3.5(0.5)$ & $5(0.04)$ & $3.96(0)$ & $112(8)$ & $-0.697(0.062)$ \\
GEMS & $7.1(0.8)$ & $2.9(0.3)$ & $1.67(0.17)$ & $0.81(0.21)$ & $199(32)$ & $-0.723(0.028)$ \\
GEOS & $7.6(0.8)$ & $3.5(0.4)$ & $2.18(0.65)$ & $1.44(0.6)$ & $155(45)$ & $-0.699(0.042)$ \\
GFDL & $7.4(1.2)$ & $3.2(0.3)$ & $1.66(0.37)$ & $0.51(0.57)$ & $267(85)$ & $-0.413(0.072)$ \\
HAD & $4.9(1)$ & $2.8(0.3)$ & $0.92(0.33)$ & $0.37(0.5)$ & $407(213)$ & $-0.601(0.071)$ \\
MIROC & $6.1(0.6)$ & $3.9(0.3)$ & $2.62(0.26)$ & $1.39(0.23)$ & $131(17)$ & $-0.77(0.023)$ \\
MPI & $9.5(1.8)$ & $3(0.3)$ & $1.24(0.21)$ & $0.38(0.31)$ & $356(125)$ & $-0.677(0.037)$ \\
NCAR & $8.2(1.2)$ & $4.1(0.5)$ & $1.27(0.08)$ & $0.61(0.11)$ & $379(72)$ & $-0.652(0.026)$ \\
\hline
\end{tabular}

JJA CAS ( Mid-N )

\begin{tabular}{rrrrrrr}
\hline & $\sigma_{P}$ & $\sigma_{T}$ & $\nu_{P}$ & $\nu_{T}$ & $a^{-1}$ & $\rho$ \\
\hline NCEP/GPCP & $4(0.6)$ & $2.1(0.2)$ & $3.34(1.85)$ & $1.69(1.16)$ & $119(54)$ & $0.071(0.101)$ \\
BCC & $2.5(0.4)$ & $3.2(0.4)$ & $5(0.13)$ & $4.31(0)$ & $102(9)$ & $-0.279(0.11)$ \\
GEMS & $6.5(1)$ & $2.8(0.2)$ & $1.83(0.21)$ & $0.9(0.26)$ & $214(38)$ & $-0.258(0.061)$ \\
GEOS & $5(0.6)$ & $3.7(0.5)$ & $1.33(0.54)$ & $1.14(0.48)$ & $224(93)$ & $-0.462(0.068)$ \\
GFDL & $4.8(0.7)$ & $2.9(0.3)$ & $2.91(1.09)$ & $1.43(0.9)$ & $125(43)$ & $-0.436(0.081)$ \\
HAD & $4.5(0.5)$ & $2.3(0.2)$ & $2.53(\mathrm{NA})$ & $1.41(1.08)$ & $143(73)$ & $0.062(0.111)$ \\
MIROC & $9.2(1.1)$ & $8.3(1)$ & $2.5(0.2)$ & $2.1(0.19)$ & $168(20)$ & $-0.9(0.013)$ \\
MPI & $6.6(1.1)$ & $3(0.3)$ & $1.4(0.2)$ & $0.71(0.28)$ & $258(64)$ & $-0.551(0.057)$ \\
NCAR & $6(0.7)$ & $3.6(0.3)$ & $1.5(0.12)$ & $0.91(0.15)$ & $191(29)$ & $-0.548(0.032)$ \\
\hline
\end{tabular}

\section{DJF CAS ( Mid-N )}


Table 10: Parameter estimates for Tibet (TIB) by season with asymptotic standard errors in parentheses

\begin{tabular}{rrrrrrrr}
\hline & $\sigma_{P}$ & $\sigma_{T}$ & $\nu_{P}$ & $\nu_{T}$ & $a^{-1}$ & $\rho$ \\
\hline NCEP/GPCP & $10.3(4.2)$ & $3.3(0.5)$ & $1.45(0.28)$ & $0.34(0.62)$ & $590(333)$ & $0.043(0.106)$ \\
BCC & $11.9(3.9)$ & $5.4(1.3)$ & $3.04(0.79)$ & $2(0.6)$ & $238(88)$ & $-0.914(0.025)$ \\
GEMS & $4.8(0.5)$ & $2.5(0.2)$ & $2.57(0.47)$ & $0.72(0.47)$ & $119(24)$ & $-0.392(0.051)$ \\
GEOS & $8.5(1.2)$ & $3.9(0.3)$ & $1.6(0.6)$ & $0.65(0.77)$ & $168(68)$ & $-0.559(0.061)$ \\
GFDL & $21.4(11.1)$ & $5.8(1.5)$ & $1.33(0.25)$ & $0.52(0.33)$ & $722(525)$ & $-0.789(0.039)$ \\
HAD & $5.6(0.9)$ & $2.4(0.2)$ & $0.93(0.4)$ & $0.14(\mathrm{NA})$ & $278(139)$ & $-0.468(0.092)$ \\
MIROC & $7.4(0.8)$ & $4.5(0.4)$ & $3.15(0.47)$ & $1.74(0.34)$ & $101(17)$ & $-0.432(0.052)$ \\
MPI & $7.7(0.8)$ & $4(0.4)$ & $4.22(1.35)$ & $2.27(0.46)$ & $79(20)$ & $-0.791(0.03)$ \\
NCAR & $7.4(0.9)$ & $3.5(0.3)$ & $1.58(0.13)$ & $0.67(0.18)$ & $180(30)$ & $-0.674(0.025)$ \\
\hline
\end{tabular}

JJA TIB ( Mid-N )

\begin{tabular}{rrrrrrrr}
\hline & $\sigma_{P}$ & $\sigma_{T}$ & $\nu_{P}$ & $\nu_{T}$ & $a^{-1}$ & $\rho$ \\
\hline NCEP/GPCP & $5.2(1)$ & $3.2(0.4)$ & $1.44(0.39)$ & $0.47(0.62)$ & $327(129)$ & $0.351(0.093)$ \\
BCC & $5.1(1.3)$ & $5(1.2)$ & $2.01(0.45)$ & $1.39(0.46)$ & $376(131)$ & $-0.762(0.06)$ \\
GEMS & $3.7(0.5)$ & $3.5(0.4)$ & $1.33(0.18)$ & $0.58(0.25)$ & $267(65)$ & $-0.049(0.064)$ \\
GEOS & $6(0.8)$ & $3.5(0.3)$ & $1.47(0.61)$ & $0.54(0.79)$ & $173(72)$ & $-0.32(0.079)$ \\
GFDL & $5.1(0.6)$ & $3.6(0.4)$ & $5(0.05)$ & $2.74(0)$ & $81(5)$ & $-0.239(0.093)$ \\
HAD & $5.2(0.6)$ & $2.7(0.3)$ & $1.2(0.92)$ & $0.55(1.23)$ & $187(113)$ & $0.002(0.129)$ \\
MIROC & $5(0.6)$ & $4.5(0.4)$ & $4.07(0.34)$ & $1.74(0.15)$ & $113(12)$ & $-0.326(0.054)$ \\
MPI & $7.1(0.8)$ & $3.4(0.3)$ & $5(0.07)$ & $1.79(0)$ & $90(4)$ & $-0.34(0.067)$ \\
NCAR & $5.6(0.6)$ & $3.8(0.3)$ & $1.67(0.17)$ & $0.86(0.2)$ & $151(25)$ & $-0.349(0.042)$ \\
\hline
\end{tabular}

\section{DJF TIB ( Mid-N )}

Table 11: Parameter estimates for Eastern Asia (EAS) by season with asymptotic standard errors in parentheses

\begin{tabular}{rrrrrrr}
\hline & $\sigma_{P}$ & $\sigma_{T}$ & $\nu_{P}$ & $\nu_{T}$ & $a^{-1}$ & $\rho$ \\
\hline NCEP/GPCP & $6.7(1.6)$ & $2.5(0.5)$ & $1.3(0.25)$ & $1.17(0.24)$ & $474(163)$ & $0.323(0.096)$ \\
BCC & $6.3(1.4)$ & $1.9(0.2)$ & $3.2(0.41)$ & $0.75(0.43)$ & $332(69)$ & $-0.157(0.096)$ \\
GEMS & $10.6(2.2)$ & $4(0.6)$ & $1.55(0.11)$ & $1.16(0.12)$ & $474(97)$ & $0.564(0.039)$ \\
GEOS & $7.3(1)$ & $2.6(0.3)$ & $0.93(0.17)$ & $0.86(0.15)$ & $419(123)$ & $-0.467(0.058)$ \\
GFDL & $5.1(0.8)$ & $2.2(0.3)$ & $1.51(0.21)$ & $0.91(0.25)$ & $370(90)$ & $-0.314(0.07)$ \\
HAD & $5.6(1)$ & $4.3(0.9)$ & $0.88(0.2)$ & $1.04(0.23)$ & $522(200)$ & $-0.207(0.098)$ \\
MIROC & $15.7(3.4)$ & $2.9(0.3)$ & $2.05(0.11)$ & $1.27(0.12)$ & $333(50)$ & $0.308(0.045)$ \\
MPI & $6(0.9)$ & $2.2(0.2)$ & $2.02(0.25)$ & $1.03(0.26)$ & $225(41)$ & $-0.199(0.066)$ \\
NCAR & $5.4(0.7)$ & $2.2(0.2)$ & $1.18(0.07)$ & $0.81(0.1)$ & $319(54)$ & $-0.002(0.038)$ \\
\hline
\end{tabular}

JJA EAS ( Mid-N )

\begin{tabular}{rrrrrrrr}
\hline & $\sigma_{P}$ & $\sigma_{T}$ & $\nu_{P}$ & $\nu_{T}$ & $a^{-1}$ & $\rho$ \\
\hline NCEP/GPCP & $6.1(0.9)$ & $3.4(0.6)$ & $1.53(0.31)$ & $1.72(0.31)$ & $338(94)$ & $0.509(0.076)$ \\
BCC & $6.4(1.1)$ & $6.3(1)$ & $3.52(0.78)$ & $2.52(0.49)$ & $273(73)$ & $0.665(0.063)$ \\
GEMS & $6.1(0.9)$ & $3.7(0.5)$ & $1.43(0.12)$ & $1.11(0.13)$ & $393(77)$ & $0.543(0.039)$ \\
GEOS & $8.1(2)$ & $6.7(1.8)$ & $0.7(0.09)$ & $0.71(0.08)$ & $1341(618)$ & $-0.408(0.063)$ \\
GFDL & $5.8(1.1)$ & $3.3(0.6)$ & $1.35(0.2)$ & $1.04(0.2)$ & $532(164)$ & $0.144(0.078)$ \\
HAD & $7.7(2.5)$ & $6.3(2.3)$ & $0.67(0.12)$ & $0.49(0.21)$ & $1650(1121)$ & $0.074(0.108)$ \\
MIROC & $8(0.9)$ & $4.8(0.6)$ & $2.22(0.14)$ & $2.11(0.13)$ & $214(24)$ & $0.786(0.021)$ \\
MPI & $6.7(1)$ & $4(0.5)$ & $1.77(0.22)$ & $1.59(0.2)$ & $306(61)$ & $0.544(0.052)$ \\
NCAR & $6.9(1.2)$ & $3.6(0.4)$ & $1.24(0.07)$ & $0.86(0.09)$ & $471(91)$ & $0.247(0.036)$ \\
\hline
\end{tabular}

\section{DJF EAS ( Mid-N )}


Table 12: Parameter estimates for Southeast Asia (SEA) by season with asymptotic standard errors in parentheses

\begin{tabular}{rrrrrrrr}
\hline & $\sigma_{P}$ & $\sigma_{T}$ & $\nu_{P}$ & $\nu_{T}$ & $a^{-1}$ & $\rho$ \\
\hline NCEP/GPCP & $4.5(0.6)$ & $1.2(0.2)$ & $3.6(1.37)$ & $5(52.56)$ & $97(15)$ & $-0.186(0.132)$ \\
BCC & $6.9(1.4)$ & $1.2(0.2)$ & $5(\mathrm{NA})$ & $2.11(0)$ & $170(23)$ & $0.19(0.164)$ \\
GEMS & $6.1(0.8)$ & $1.2(0.1)$ & $1.06(0.27)$ & $0.72(0.31)$ & $236(79)$ & $-0.011(0.084)$ \\
GEOS & $9.8(2)$ & $1.1(0.1)$ & $0.85(0.25)$ & $0.14(1.75)$ & $511(287)$ & $-0.458(0.092)$ \\
GFDL & $6.1(1.4)$ & $1.1(0.1)$ & $1.99(0.57)$ & $0.55(0.73)$ & $351(143)$ & $-0.161(0.13)$ \\
HAD & $5.7(0.9)$ & $1.2(0.1)$ & $2.77(\mathrm{NA})$ & $0.18(36.12)$ & $168(103)$ & $0.331(0.144)$ \\
MIROC & $5.9(0.6)$ & $1.5(0.2)$ & $1.66(0.78)$ & $1.59(0.79)$ & $131(55)$ & $-0.38(0.071)$ \\
MPI & $6.5(0.8)$ & $1(0.1)$ & $0.68(0.4)$ & $0.5(0.42)$ & $275(164)$ & $0.264(0.101)$ \\
NCAR & $5(0.6)$ & $1.3(0.1)$ & $1.02(0.14)$ & $0.38(0.25)$ & $290(79)$ & $-0.434(0.049)$ \\
\hline
\end{tabular}

JJA SEA ( Equat )

\begin{tabular}{rrrrrrrr}
\hline & $\sigma_{P}$ & $\sigma_{T}$ & $\nu_{P}$ & $\nu_{T}$ & $a^{-1}$ & $\rho$ \\
\hline NCEP/GPCP & $6.6(1.3)$ & $1.7(0.4)$ & $1.34(0.36)$ & $1.15(0.4)$ & $512(212)$ & $-0.051(0.143)$ \\
BCC & $11.1(3.2)$ & $2.4(0.6)$ & $1.12(0.4)$ & $0.86(0.38)$ & $721(388)$ & $-0.545(0.13)$ \\
GEMS & $7.4(1.8)$ & $1.9(0.3)$ & $0.52(0.08)$ & $0.37(0.11)$ & $1229(697)$ & $-0.225(0.078)$ \\
GEOS & $12.4(3.4)$ & $2(0.4)$ & $0.73(0.17)$ & $0.33(0.27)$ & $1009(615)$ & $-0.533(0.089)$ \\
GFDL & $11.7(2.7)$ & $1.9(0.4)$ & $1.6(0.37)$ & $0.79(0.37)$ & $606(219)$ & $-0.262(0.124)$ \\
HAD & $6.1(1.2)$ & $2.3(0.3)$ & $2.09(0.35)$ & $1.88(\mathrm{NA})$ & $421(204)$ & $0.255(0.154)$ \\
MIROC & $10.7(2)$ & $1.7(0.2)$ & $1.49(0.34)$ & $0.76(0.32)$ & $318(101)$ & $-0.37(0.076)$ \\
MPI & $9.6(2)$ & $1.4(0.2)$ & $0.42(0.13)$ & $0.26(0.14)$ & $1373(838)$ & $0.146(0.101)$ \\
NCAR & $12.8(2.8)$ & $2.7(0.4)$ & $1.12(0.1)$ & $0.57(0.15)$ & $683(199)$ & $-0.545(0.047)$ \\
\hline
\end{tabular}

DJF SEA ( Equat ) 
Table 13: Parameter estimates for the Sahara (SAH) by season with asymptotic standard errors in parentheses

\begin{tabular}{rrrrrrr}
\hline & $\sigma_{P}$ & $\sigma_{T}$ & $\nu_{P}$ & $\nu_{T}$ & $a^{-1}$ & $\rho$ \\
\hline NCEP/GPCP & $12.9(5.1)$ & $9.7(4.2)$ & $1.4(0.15)$ & $1.64(0.14)$ & $1077(444)$ & $-0.245(0.072)$ \\
BCC & $4.4(0.8)$ & $3.1(0.5)$ & $2.88(0.43)$ & $2.12(0.36)$ & $309(63)$ & $-0.336(0.073)$ \\
GEMS & $10.9(4)$ & $11.3(5.1)$ & $1(0.07)$ & $1.13(0.06)$ & $1528(705)$ & $-0.272(0.042)$ \\
GEOS & $11.7(4)$ & $5.1(1.5)$ & $1.4(0.15)$ & $1.02(0.16)$ & $889(356)$ & $-0.499(0.05)$ \\
GFDL & $9.8(1.9)$ & $2.8(0.4)$ & $1.77(0.2)$ & $1.04(0.21)$ & $488(118)$ & $-0.247(0.062)$ \\
HAD & $7.6(1.5)$ & $5.2(1.2)$ & $0.97(0.2)$ & $0.97(0.17)$ & $851(353)$ & $-0.647(0.05)$ \\
MIROC & $7.8(1.3)$ & $11.9(2.9)$ & $1.64(0.11)$ & $2.29(0.1)$ & $444(78)$ & $-0.437(0.038)$ \\
MPI & $8.4(2)$ & $7.8(2.5)$ & $1.17(0.16)$ & $1.54(0.13)$ & $600(217)$ & $-0.375(0.05)$ \\
NCAR & $9.7(3)$ & $4.7(1.1)$ & $1.16(0.06)$ & $0.86(0.07)$ & $864(288)$ & $-0.163(0.033)$ \\
\hline
\end{tabular}

JJA SAH ( Equat )

\begin{tabular}{rrrrrrrr}
\hline & $\sigma_{P}$ & $\sigma_{T}$ & $\nu_{P}$ & $\nu_{T}$ & $a^{-1}$ & $\rho$ \\
\hline NCEP/GPCP & $5(1.1)$ & $2(0.4)$ & $1.02(0.13)$ & $0.76(0.14)$ & $933(293)$ & $-0.235(0.078)$ \\
BCC & $3(0.6)$ & $2.5(0.5)$ & $1.15(0.19)$ & $0.92(0.19)$ & $659(214)$ & $-0.483(0.065)$ \\
GEMS & $5.9(0.9)$ & $2.7(0.3)$ & $1.47(0.12)$ & $1.06(0.11)$ & $430(81)$ & $-0.144(0.045)$ \\
GEOS & $4.1(0.8)$ & $4.8(1)$ & $1.2(0.14)$ & $1.29(0.17)$ & $569(162)$ & $-0.207(0.067)$ \\
GFDL & $4.1(0.9)$ & $2.9(0.5)$ & $0.99(0.16)$ & $0.73(0.15)$ & $697(237)$ & $-0.345(0.064)$ \\
HAD & $3(0.3)$ & $2(0.2)$ & $0.91(0.33)$ & $0.55(0.51)$ & $406(174)$ & $0.269(0.084)$ \\
MIROC & $5.3(0.8)$ & $1.9(0.2)$ & $2.27(0.13)$ & $0.96(0.14)$ & $367(49)$ & $-0.171(0.043)$ \\
MPI & $3.3(0.4)$ & $2.7(0.4)$ & $0.67(0.1)$ & $0.7(0.1)$ & $708(211)$ & $-0.117(0.058)$ \\
NCAR & $5.5(1)$ & $3.6(0.5)$ & $1.27(0.06)$ & $0.83(0.07)$ & $590(110)$ & $-0.581(0.022)$ \\
\hline
\end{tabular}

DJF SAH ( Equat ) 
Table 14: Parameter estimates for West Africa (WAF) by season with asymptotic standard errors in parentheses

\begin{tabular}{rrrrrrrr}
\hline & $\sigma_{P}$ & $\sigma_{T}$ & $\nu_{P}$ & $\nu_{T}$ & $a^{-1}$ & $\rho$ \\
\hline NCEP/GPCP & $13.3(4.1)$ & $3.1(1)$ & $2.12(0.38)$ & $2.11(0.35)$ & $510(183)$ & $-0.249(0.118)$ \\
BCC & $30.3(19.3)$ & $4.1(2.2)$ & $1.5(0.22)$ & $1.04(0.29)$ & $1508(803)$ & $0.122(0.215)$ \\
GEMS & $14.3(3.8)$ & $2(0.4)$ & $1.73(0.16)$ & $1.03(0.14)$ & $574(154)$ & $0.147(0.068)$ \\
GEOS & $13.1(3.7)$ & $2.5(0.7)$ & $0.78(0.12)$ & $0.7(0.13)$ & $1039(560)$ & $-0.538(0.07)$ \\
GFDL & $11(4.6)$ & $1.6(0.4)$ & $0.96(0.15)$ & $0.46(0.25)$ & $1239(775)$ & $0.054(0.114)$ \\
HAD & $11(4.4)$ & $3.4(1.9)$ & $0.71(0.12)$ & $0.94(0.12)$ & $2057(1503)$ & $-0.553(0.09)$ \\
MIROC & $24.4(8.1)$ & $3.9(1.2)$ & $1.54(0.13)$ & $1.22(0.12)$ & $764(268)$ & $-0.385(0.054)$ \\
MPI & $18.5(6.2)$ & $4.3(1.5)$ & $1.42(0.2)$ & $1.29(0.2)$ & $751(322)$ & $0.085(0.093)$ \\
NCAR & $18.7(6.6)$ & $4(1.3)$ & $1.12(0.07)$ & $0.87(0.08)$ & $1310(507)$ & $0.199(0.048)$ \\
\hline
\end{tabular}

JJA WAF ( Equat )

\begin{tabular}{rrrrrrrr}
\hline & $\sigma_{P}$ & $\sigma_{T}$ & $\nu_{P}$ & $\nu_{T}$ & $a^{-1}$ & $\rho$ \\
\hline NCEP/GPCP & $6.3(1.7)$ & $2.2(0.7)$ & $1.54(0.51)$ & $2.03(0.43)$ & $369(164)$ & $0.4(0.12)$ \\
BCC & $7.8(2.4)$ & $1.7(0.4)$ & $1.62(0.34)$ & $0.94(0.36)$ & $522(201)$ & $-0.147(0.15)$ \\
GEMS & $8.5(2)$ & $1.3(0.2)$ & $1.68(0.17)$ & $0.63(0.21)$ & $416(106)$ & $0.212(0.065)$ \\
GEOS & $6.1(1.4)$ & $2.6(0.6)$ & $0.7(0.15)$ & $0.79(0.14)$ & $657(308)$ & $0.042(0.102)$ \\
GFDL & $6.5(1.6)$ & $1.2(0.2)$ & $1.36(0.28)$ & $0.47(0.42)$ & $438(172)$ & $-0.167(0.103)$ \\
HAD & $3.9(0.6)$ & $1.2(0.2)$ & $0.81(0.32)$ & $0.63(0.52)$ & $420(230)$ & $-0.094(0.131)$ \\
MIROC & $6.9(1.2)$ & $1.8(0.3)$ & $1.34(0.15)$ & $1.06(0.16)$ & $336(81)$ & $0.303(0.058)$ \\
MPI & $7.2(1.2)$ & $1.6(0.2)$ & $2.1(0.48)$ & $1.32(0.41)$ & $215(61)$ & $0.613(0.059)$ \\
NCAR & $9.7(2.4)$ & $2.3(0.4)$ & $1.25(0.08)$ & $0.78(0.1)$ & $512(138)$ & $0.275(0.045)$ \\
\hline
\end{tabular}

\section{DJF WAF ( Equat )}


Table 15: Parameter estimates for East Africa (EAF) by season with asymptotic standard errors in parentheses

\begin{tabular}{rrrrrrr}
\hline & $\sigma_{P}$ & $\sigma_{T}$ & $\nu_{P}$ & $\nu_{T}$ & $a^{-1}$ & $\rho$ \\
\hline NCEP/GPCP & $11.9(3.3)$ & $2.1(0.4)$ & $1.35(0.24)$ & $0.85(0.27)$ & $590(232)$ & $0.014(0.104)$ \\
BCC & $10.1(2.8)$ & $1.7(0.2)$ & $3.18(0.67)$ & $0.97(0.48)$ & $329(100)$ & $-0.127(0.117)$ \\
GEMS & $9(1.7)$ & $1.7(0.2)$ & $1.61(0.14)$ & $0.56(0.22)$ & $414(91)$ & $-0.237(0.054)$ \\
GEOS & $12.6(1.9)$ & $2(0.2)$ & $1.62(0.3)$ & $0.8(0.37)$ & $316(89)$ & $-0.609(0.051)$ \\
GFDL & $8.8(1.4)$ & $2.5(0.3)$ & $1.82(0.38)$ & $1.09(0.39)$ & $286(81)$ & $-0.428(0.074)$ \\
HAD & $9.4(1.7)$ & $1.9(0.2)$ & $0.92(0.27)$ & $0.3(0.59)$ & $546(259)$ & $-0.666(0.061)$ \\
MIROC & $10.3(1.1)$ & $2.6(0.3)$ & $2.11(0.21)$ & $1.56(0.19)$ & $193(28)$ & $-0.752(0.025)$ \\
MPI & $10.2(1.8)$ & $1.9(0.3)$ & $0.77(0.13)$ & $0.41(0.16)$ & $717(293)$ & $-0.451(0.059)$ \\
NCAR & $6.1(0.5)$ & $1.9(0.1)$ & $1.93(0.18)$ & $1.03(0.19)$ & $169(24)$ & $-0.095(0.041)$ \\
\hline
\end{tabular}

JJA EAF ( Equat )

\begin{tabular}{rrrrrrrr}
\hline & $\sigma_{P}$ & $\sigma_{T}$ & $\nu_{P}$ & $\nu_{T}$ & $a^{-1}$ & $\rho$ \\
\hline NCEP/GPCP & $4.9(0.9)$ & $1.5(0.2)$ & $1.59(0.39)$ & $0.83(0.39)$ & $354(127)$ & $-0.608(0.06)$ \\
BCC & $6.6(1.4)$ & $2.2(0.4)$ & $2.41(0.44)$ & $1.47(0.4)$ & $337(88)$ & $-0.706(0.061)$ \\
GEMS & $5.4(1)$ & $2.4(0.4)$ & $1.11(0.11)$ & $0.72(0.13)$ & $517(139)$ & $-0.433(0.05)$ \\
GEOS & $7.5(1.2)$ & $2.5(0.3)$ & $1.97(0.45)$ & $1.41(0.43)$ & $248(73)$ & $-0.392(0.071)$ \\
GFDL & $4.8(0.7)$ & $2(0.2)$ & $1.88(0.42)$ & $1.02(0.44)$ & $242(67)$ & $-0.587(0.057)$ \\
HAD & $5.5(1.2)$ & $1.9(0.3)$ & $0.86(0.19)$ & $0.41(0.41)$ & $712(327)$ & $-0.215(0.116)$ \\
MIROC & $6.6(0.6)$ & $2.4(0.2)$ & $2.13(0.26)$ & $2.1(0.26)$ & $130(17)$ & $-0.808(0.021)$ \\
MPI & $6.1(1)$ & $2.2(0.4)$ & $0.58(0.1)$ & $0.55(0.1)$ & $708(288)$ & $-0.28(0.071)$ \\
NCAR & $5.7(0.6)$ & $2.4(0.2)$ & $1.73(0.1)$ & $1.01(0.12)$ & $249(32)$ & $-0.446(0.033)$ \\
\hline
\end{tabular}

DJF EAF ( Equat )

Table 16: Parameter estimates for South Africa (SAF) by season with asymptotic standard errors in parentheses

\begin{tabular}{rrrrrrrr}
\hline & $\sigma_{P}$ & $\sigma_{T}$ & $\nu_{P}$ & $\nu_{T}$ & $a^{-1}$ & $\rho$ \\
\hline NCEP/GPCP & $11.9(4.8)$ & $2(0.5)$ & $1.51(0.4)$ & $1.14(0.37)$ & $480(201)$ & $0.64(0.103)$ \\
BCC & $7(1.7)$ & $2.7(0.6)$ & $2.23(0.57)$ & $1.71(0.49)$ & $356(124)$ & $0.473(0.102)$ \\
GEMS & $5.9(1.6)$ & $2.1(0.4)$ & $1.14(0.13)$ & $0.58(0.19)$ & $648(236)$ & $0.191(0.066)$ \\
GEOS & $9.8(1.8)$ & $2.1(0.3)$ & $1.87(0.56)$ & $1.29(0.52)$ & $235(87)$ & $-0.491(0.075)$ \\
GFDL & $8.8(2.1)$ & $1.8(0.3)$ & $1.7(0.45)$ & $1.19(0.43)$ & $308(113)$ & $-0.107(0.131)$ \\
HAD & $3.6(0.5)$ & $2.2(0.4)$ & $0.7(0.38)$ & $0.97(0.38)$ & $318(164)$ & $-0.064(0.133)$ \\
MIROC & $13.4(2.9)$ & $2.5(0.4)$ & $1.92(0.15)$ & $1.11(0.19)$ & $357(73)$ & $0.721(0.033)$ \\
MPI & $5.5(1.1)$ & $1.4(0.1)$ & $3.02(0.96)$ & $0.94(0.68)$ & $154(51)$ & $-0.002(0.086)$ \\
NCAR & $8.4(1.6)$ & $2.2(0.3)$ & $1.37(0.12)$ & $0.76(0.15)$ & $362(91)$ & $-0.036(0.05)$ \\
\hline
\end{tabular}

JJA SAF ( Mid-S )

\begin{tabular}{rrrrrrr}
\hline & $\sigma_{P}$ & $\sigma_{T}$ & $\nu_{P}$ & $\nu_{T}$ & $a^{-1}$ & $\rho$ \\
\hline NCEP/GPCP & $6.7(1.2)$ & $3.1(0.8)$ & $2.07(0.42)$ & $2.9(0.46)$ & $317(78)$ & $0.114(0.125)$ \\
BCC & $8.9(1.6)$ & $2.8(0.6)$ & $4.81(0.5)$ & $2.93(0.11)$ & $216(30)$ & $-0.389(0.112)$ \\
GEMS & $12.4(4.4)$ & $3.8(1.2)$ & $1.28(0.13)$ & $0.98(0.14)$ & $802(335)$ & $-0.134(0.068)$ \\
GEOS & $14.2(3.8)$ & $6.4(2)$ & $1.27(0.21)$ & $1.28(0.18)$ & $655(258)$ & $-0.61(0.063)$ \\
GFDL & $17.8(6.1)$ & $3.6(1)$ & $1.89(0.26)$ & $1.07(0.27)$ & $661(240)$ & $-0.629(0.074)$ \\
HAD & $6.5(1.2)$ & $3.4(1.1)$ & $3.3(1.83)$ & $3.75($ NA $)$ & $160(89)$ & $-0.398(0.134)$ \\
MIROC & $14.1(3.2)$ & $8.4(2.5)$ & $1.65(0.16)$ & $1.86(0.13)$ & $502(129)$ & $-0.47(0.051)$ \\
MPI & $10.4(2.9)$ & $4.9(2)$ & $1.05(0.2)$ & $1.15(0.17)$ & $646(295)$ & $-0.4(0.074)$ \\
NCAR & $12.4(3.3)$ & $4.2(1)$ & $1.45(0.12)$ & $1.03(0.11)$ & $522(157)$ & $-0.425(0.043)$ \\
\hline
\end{tabular}

DJF SAF ( Mid-S ) 
Table 17: Parameter estimates for Central America (CAM) by season with asymptotic standard errors in parentheses

\begin{tabular}{rrrrrrrr}
\hline & $\sigma_{P}$ & $\sigma_{T}$ & $\nu_{P}$ & $\nu_{T}$ & $a^{-1}$ & $\rho$ \\
\hline NCEP/GPCP & $6.7(1.6)$ & $1.7(0.3)$ & $1.5(0.82)$ & $0.79(0.84)$ & $254(144)$ & $0.103(0.169)$ \\
BCC & $6.7(1.3)$ & $1.6(0.3)$ & $4(\mathrm{NA})$ & $2.42(2.49)$ & $120(96)$ & $-0.707(0.102)$ \\
GEMS & $5.7(0.8)$ & $2.1(0.3)$ & $1.59(0.51)$ & $1.08(0.52)$ & $200(81)$ & $-0.342(0.091)$ \\
GEOS & $8.8(1.5)$ & $2.8(0.4)$ & $2.25(1.18)$ & $1.38(0.97)$ & $170(79)$ & $-0.336(0.122)$ \\
GFDL & $11.6(2.9)$ & $2.7(0.4)$ & $0.96(0.42)$ & $0.46(0.56)$ & $332(187)$ & $-0.673(0.097)$ \\
HAD & $7.6(2.9)$ & $2.3(0.3)$ & $2.22(\mathrm{NA})$ & $0.92(21.79)$ & $541(352)$ & $-0.02(0.177)$ \\
MIROC & $7.9(1.1)$ & $2.2(0.2)$ & $2.96(0.87)$ & $1.9(0.66)$ & $107(31)$ & $-0.553(0.064)$ \\
MPI & $6.3(1)$ & $1.7(0.2)$ & $1.82(0.95)$ & $0.59(1.04)$ & $186(103)$ & $-0.303(0.104)$ \\
NCAR & $9.1(1.3)$ & $2.4(0.2)$ & $2.02(0.37)$ & $0.95(0.35)$ & $147(37)$ & $-0.493(0.055)$ \\
\hline
\end{tabular}

JJA CAM ( Mid-N )

\begin{tabular}{rrrrrrrr}
\hline & $\sigma_{P}$ & $\sigma_{T}$ & $\nu_{P}$ & $\nu_{T}$ & $a^{-1}$ & $\rho$ \\
\hline NCEP/GPCP & $3.6(0.4)$ & $1.6(0.3)$ & $0.1(\mathrm{NA})$ & $0.84(0)$ & $261(223)$ & $0.263(0.156)$ \\
BCC & $6(1.3)$ & $2.8(0.6)$ & $5(\mathrm{NA})$ & $4.76(0.02)$ & $113(19)$ & $-0.434(0.171)$ \\
GEMS & $6(1)$ & $2.5(0.3)$ & $1.81(0.65)$ & $1.11(0.59)$ & $184(78)$ & $0.156(0.1)$ \\
GEOS & $8.2(1.4)$ & $4.5(1.1)$ & $0.43(0.22)$ & $0.71(0.16)$ & $576(420)$ & $-0.671(0.075)$ \\
GFDL & $7.5(1.8)$ & $2.6(0.3)$ & $3.03(2.09)$ & $0.97(1.28)$ & $160(98)$ & $-0.11(0.153)$ \\
HAD & $5(1)$ & $3.1(0.7)$ & $2.22(\mathrm{NA})$ & $0.37(0.99)$ & $254(153)$ & $-0.142(0.193)$ \\
MIROC & $5.6(0.9)$ & $2.1(0.2)$ & $2.5(0.47)$ & $1.04(0.45)$ & $167(43)$ & $0.087(0.086)$ \\
MPI & $5.9(1)$ & $1.9(0.2)$ & $1.9(0.93)$ & $1.04(0.86)$ & $155(72)$ & $0(0.116)$ \\
NCAR & $9.2(2.4)$ & $3(0.4)$ & $1.02(0.13)$ & $0.55(0.19)$ & $443(183)$ & $-0.28(0.063)$ \\
\hline
\end{tabular}

\section{DJF CAM ( Mid-N )}


Table 18: Parameter estimates for the Amazon (AMZ) by season with asymptotic standard errors in parentheses

\begin{tabular}{rrrrrrrr}
\hline & $\sigma_{P}$ & $\sigma_{T}$ & $\nu_{P}$ & $\nu_{T}$ & $a^{-1}$ & $\rho$ \\
\hline NCEP/GPCP & $11.7(3.9)$ & $1.9(0.2)$ & $1.21(0.17)$ & $0.25(0.41)$ & $843(364)$ & $-0.232(0.073)$ \\
BCC & $21.4(6.4)$ & $4.7(0.8)$ & $2.31(0.38)$ & $1.28(0.35)$ & $485(155)$ & $-0.842(0.03)$ \\
GEMS & $11.6(4.3)$ & $3.7(1.1)$ & $0.85(0.08)$ & $0.64(0.1)$ & $931(534)$ & $0.062(0.05)$ \\
GEOS & $10.4(1.6)$ & $3(0.3)$ & $0.78(0.13)$ & $0.44(0.21)$ & $508(193)$ & $-0.626(0.041)$ \\
GFDL & $17.7(5.1)$ & $5(0.9)$ & $1.17(0.14)$ & $0.65(0.17)$ & $825(335)$ & $-0.843(0.021)$ \\
HAD & $8(1.7)$ & $2.7(0.4)$ & $0.89(0.17)$ & $0.46(0.37)$ & $689(308)$ & $-0.195(0.094)$ \\
MIROC & $9.9(0.9)$ & $2.9(0.2)$ & $2.35(0.24)$ & $1.45(0.24)$ & $155(20)$ & $-0.596(0.029)$ \\
MPI & $10.9(1.5)$ & $2.7(0.2)$ & $1.39(0.19)$ & $0.55(0.25)$ & $359(85)$ & $-0.641(0.034)$ \\
NCAR & $6.8(0.5)$ & $2.9(0.2)$ & $1.43(0.1)$ & $0.9(0.12)$ & $212(28)$ & $-0.512(0.025)$ \\
\hline
\end{tabular}

JJA AMZ ( Equat )

\begin{tabular}{rrrrrrr}
\hline & $\sigma_{P}$ & $\sigma_{T}$ & $\nu_{P}$ & $\nu_{T}$ & $a^{-1}$ & $\rho$ \\
\hline NCEP/GPCP & $12.5(4.3)$ & $1.4(0.1)$ & $1.24(0.24)$ & $0.17(0.92)$ & $685(305)$ & $0.305(0.084)$ \\
BCC & $9.2(1.3)$ & $1.9(0.2)$ & $4.67(0.56)$ & $1.49(0.11)$ & $204(27)$ & $-0.578(0.051)$ \\
GEMS & $12.4(3.2)$ & $2.6(0.4)$ & $0.95(0.11)$ & $0.54(0.16)$ & $562(253)$ & $-0.056(0.048)$ \\
GEOS & $10.9(2.1)$ & $2.8(0.3)$ & $0.74(0.11)$ & $0.35(0.2)$ & $716(308)$ & $-0.624(0.041)$ \\
GFDL & $11.6(2.2)$ & $2.4(0.2)$ & $1.73(0.24)$ & $0.47(0.38)$ & $379(98)$ & $-0.622(0.04)$ \\
HAD & $11.2(2.9)$ & $2(0.2)$ & $0.86(0.29)$ & $0.19(0.99)$ & $627(403)$ & $0.038(0.095)$ \\
MIROC & $9.5(0.9)$ & $2.2(0.1)$ & $2.68(0.28)$ & $1.19(0.27)$ & $143(19)$ & $-0.318(0.04)$ \\
MPI & $10.5(2)$ & $2(0.1)$ & $1.07(0.16)$ & $0.1(16.98)$ & $477(156)$ & $-0.259(0.053)$ \\
NCAR & $8.3(0.8)$ & $2.7(0.3)$ & $1.35(0.12)$ & $0.91(0.14)$ & $230(41)$ & $-0.152(0.034)$ \\
\hline
\end{tabular}

\section{DJF AMZ ( Equat )}


Table 19: Parameter estimates for Southern South America (SSA) by season with asymptotic standard errors in parentheses

\begin{tabular}{rrrrrrr}
\hline & $\sigma_{P}$ & $\sigma_{T}$ & $\nu_{P}$ & $\nu_{T}$ & $a^{-1}$ & $\rho$ \\
\hline NCEP/GPCP & $8.6(1.7)$ & $2.2(0.4)$ & $1.68(0.53)$ & $0.85(0.67)$ & $322(143)$ & $0.467(0.094)$ \\
BCC & $5(1)$ & $2.1(0.3)$ & $5(0.12)$ & $2.47(0)$ & $166(14)$ & $-0.129(0.135)$ \\
GEMS & $8.9(1.5)$ & $3.3(0.6)$ & $2.38(0.27)$ & $1.46(0.27)$ & $233(47)$ & $0.338(0.064)$ \\
GEOS & $9.5(2)$ & $8(3.3)$ & $0.7(0.15)$ & $1.2(0.13)$ & $625(318)$ & $-0.539(0.074)$ \\
GFDL & $6.4(1.2)$ & $3(0.5)$ & $3.43(1.45)$ & $2.08(0.96)$ & $155(65)$ & $-0.473(0.09)$ \\
HAD & $10.4(2.9)$ & $2.3(0.3)$ & $3.26(1.46)$ & $0.74(1.16)$ & $212(99)$ & $-0.068(0.154)$ \\
MIROC & $9.9(1.9)$ & $2(0.2)$ & $2.68(0.38)$ & $0.43(0.58)$ & $228(56)$ & $0.125(0.066)$ \\
MPI & $8.6(1.7)$ & $2(0.2)$ & $1.52(0.34)$ & $0.42(0.66)$ & $305(121)$ & $0.262(0.081)$ \\
NCAR & $5.8(0.7)$ & $2.7(0.2)$ & $2.26(0.21)$ & $1.45(0.2)$ & $141(21)$ & $0.01(0.051)$ \\
\hline
\end{tabular}

JJA SSA ( Mid-S )

\begin{tabular}{rrrrrrrr}
\hline \multicolumn{1}{c}{$\sigma_{P}$} & $\sigma_{T}$ & $\nu_{P}$ & $\nu_{T}$ & $a^{-1}$ & $\rho$ \\
\hline NCEP/GPCP & $10.3(2.8)$ & $3.5(0.8)$ & $1.35(0.44)$ & $1.14(0.5)$ & $358(186)$ & $0.558(0.089)$ \\
BCC & $10.6(2.5)$ & $4.1(1.1)$ & $3.63(1.33)$ & $2.83(0.97)$ & $203(70)$ & $0.555(0.139)$ \\
GEMS & $11.7(1.8)$ & $3(0.4)$ & $3.25(0.37)$ & $1.86(0.29)$ & $155(26)$ & $0.301(0.067)$ \\
GEOS & $16.1(4.2)$ & $3.7(0.6)$ & $1.3(0.28)$ & $0.91(0.3)$ & $397(172)$ & $-0.72(0.049)$ \\
GFDL & $10.1(1.5)$ & $3.5(0.4)$ & $4.36(0.69)$ & $1.91(0.28)$ & $147(26)$ & $-0.618(0.063)$ \\
HAD & $9(2.1)$ & $2.8(0.4)$ & $2.45(1.08)$ & $1.31(1.09)$ & $212(99)$ & $-0.519(0.109)$ \\
MIROC & $7.7(0.9)$ & $3.4(0.4)$ & $3.89(0.69)$ & $1.93(0.33)$ & $100(18)$ & $-0.054(0.072)$ \\
MPI & $9(1)$ & $3.3(0.3)$ & $2.67(1.16)$ & $1.99(0.99)$ & $117(43)$ & $-0.251(0.1)$ \\
NCAR & $10.9(1.5)$ & $2.2(0.2)$ & $3.43(0.32)$ & $1.5(0.22)$ & $109(15)$ & $-0.094(0.051)$ \\
\hline
\end{tabular}

DJF SSA ( Mid-S ) 
Table 20: Parameter estimates for Australia (AUS) by season with asymptotic standard errors in parentheses

\begin{tabular}{rrrrrrr}
\hline & $\sigma_{P}$ & $\sigma_{T}$ & $\nu_{P}$ & $\nu_{T}$ & $a^{-1}$ & $\rho$ \\
\hline NCEP/GPCP & $18.1(11)$ & $9(5.8)$ & $1.06(0.13)$ & $1.03(0.17)$ & $3405(2241)$ & $-0.103(0.106)$ \\
BCC & $7.9(4.2)$ & $5.1(2)$ & $1.34(0.21)$ & $1.12(0.21)$ & $1118(598)$ & $-0.131(0.123)$ \\
GEMS & $30.1(20.8)$ & $6(2.9)$ & $1.05(0.1)$ & $0.77(0.09)$ & $4929(3165)$ & $0.093(0.063)$ \\
GEOS & $44.8(23.9)$ & $4.2(1.3)$ & $2.26(0.22)$ & $1.16(0.29)$ & $785(281)$ & $-0.565(0.083)$ \\
GFDL & $23.5(15.5)$ & $3.3(1.3)$ & $1.65(0.22)$ & $0.95(0.2)$ & $1306(678)$ & $-0.362(0.102)$ \\
HAD & $3.2(0.9)$ & $2.4(0.5)$ & $1.47(1.03)$ & $1.42(0.98)$ & $288(209)$ & $0.436(0.1)$ \\
MIROC & $23.2(11.7)$ & $2.8(0.7)$ & $1.72(0.1)$ & $0.82(0.15)$ & $1131(423)$ & $-0.38(0.052)$ \\
MPI & $3.2(0.6)$ & $1.3(0.2)$ & $3.06(0.61)$ & $1.88(0.47)$ & $176(46)$ & $-0.211(0.076)$ \\
NCAR & $50.7(29.4)$ & $6.9(2.5)$ & $1.5(0.07)$ & $0.91(0.09)$ & $1770(775)$ & $-0.625(0.029)$ \\
\hline
\end{tabular}

JJA AUS ( Mid-S )

\begin{tabular}{rrrrrrrr}
\hline & $\sigma_{P}$ & $\sigma_{T}$ & $\nu_{P}$ & $\nu_{T}$ & $a^{-1}$ & $\rho$ \\
\hline NCEP/GPCP & $6.5(1.4)$ & $6.5(2.2)$ & $1.98(0.27)$ & $2.85(0.37)$ & $503(140)$ & $-0.162(0.1)$ \\
BCC & $10.7(4)$ & $5.3(2.1)$ & $1.59(0.22)$ & $1.46(0.2)$ & $1057(419)$ & $-0.127(0.109)$ \\
GEMS & $15(7.7)$ & $5.6(2.3)$ & $1.17(0.08)$ & $0.96(0.07)$ & $2350(1080)$ & $0.027(0.062)$ \\
GEOS & $7.4(1.7)$ & $7(2)$ & $1.28(0.18)$ & $1.5(0.19)$ & $645(221)$ & $-0.496(0.065)$ \\
GFDL & $8.1(3)$ & $5.6(2.2)$ & $1.15(0.12)$ & $1.2(0.12)$ & $1452(658)$ & $-0.645(0.053)$ \\
HAD & $6.7(2.3)$ & $12.2(6)$ & $0.91(0.14)$ & $1.43(0.13)$ & $1668(846)$ & $-0.574(0.084)$ \\
MIROC & $11(2.9)$ & $5.3(1.2)$ & $1.97(0.13)$ & $1.93(0.11)$ & $485(94)$ & $-0.234(0.053)$ \\
MPI & $5.6(1.2)$ & $6.3(1.9)$ & $1.47(0.18)$ & $2.14(0.18)$ & $529(144)$ & $-0.5(0.059)$ \\
NCAR & $11.4(4.3)$ & $6.6(2.3)$ & $1.3(0.07)$ & $1.14(0.07)$ & $1159(403)$ & $-0.075(0.045)$ \\
\hline
\end{tabular}

\section{DJF AUS ( Mid-S )}


Table 21: Parameter estimates for Southern Asia (SAS) by season with asymptotic standard errors in parentheses

\begin{tabular}{rrrrrrrr}
\hline & $\sigma_{P}$ & $\sigma_{T}$ & $\nu_{P}$ & $\nu_{T}$ & $a^{-1}$ & $\rho$ \\
\hline NCEP/GPCP & $8.4(1.3)$ & $3.1(0.4)$ & $4.99(0.27)$ & $3.1(0)$ & $109(11)$ & $-0.52(0.102)$ \\
BCC & $12.8(2.3)$ & $4.9(0.9)$ & $5(\mathrm{NA})$ & $3.52(0)$ & $154(12)$ & $-0.918(0.024)$ \\
GEMS & $11.7(2.3)$ & $2.4(0.2)$ & $1.37(0.21)$ & $0.39(0.37)$ & $321(100)$ & $-0.489(0.057)$ \\
GEOS & $16(3.6)$ & $2.8(0.3)$ & $1.62(0.47)$ & $0.53(0.62)$ & $310(133)$ & $-0.214(0.105)$ \\
GFDL & $10.2(1.7)$ & $3(0.3)$ & $5(\mathrm{NA})$ & $1.74(0)$ & $113(9)$ & $-0.537(0.087)$ \\
HAD & $9.6(2.1)$ & $2.9(0.5)$ & $2.37(0.42)$ & $2.1(0.5)$ & $313(130)$ & $-0.475(0.117)$ \\
MIROC & $14.4(1.6)$ & $3.7(0.4)$ & $4.99(0.12)$ & $3.03(0)$ & $86(4)$ & $-0.668(0.044)$ \\
MPI & $13(2.1)$ & $3.4(0.4)$ & $5(0.01)$ & $3.06(0)$ & $88(7)$ & $-0.619(0.064)$ \\
NCAR & $8.4(0.8)$ & $2.4(0.2)$ & $1.94(0.46)$ & $1.17(0.45)$ & $103(27)$ & $-0.58(0.037)$ \\
\hline
\end{tabular}

JJA SAS ( Equat )

\begin{tabular}{rrrrrrrr}
\hline & $\sigma_{P}$ & $\sigma_{T}$ & $\nu_{P}$ & $\nu_{T}$ & $a^{-1}$ & $\rho$ \\
\hline NCEP/GPCP & $3.8(0.8)$ & $2.9(0.4)$ & $3.58(1.97)$ & $1.65(1.17)$ & $149(75)$ & $-0.158(0.134)$ \\
BCC & $6.4(6.4)$ & $4.6(6.3)$ & $4.86(\mathrm{NA})$ & $3.34(2.72)$ & $161(331173)$ & $-0.729(2.156)$ \\
GEMS & $7.3(1.8)$ & $2.2(0.3)$ & $1.58(0.24)$ & $0.47(0.38)$ & $349(117)$ & $-0.33(0.071)$ \\
GEOS & $8.1(1.8)$ & $2.7(0.2)$ & $1.81(0.75)$ & $0.28(1.58)$ & $237(123)$ & $-0.364(0.095)$ \\
GFDL & $7.4(2)$ & $3.6(0.6)$ & $2.74(0.73)$ & $1.15(0.73)$ & $198(70)$ & $-0.709(0.066)$ \\
HAD & $4.2(0.6)$ & $1.8(0.2)$ & $4.98(0.73)$ & $0.19(\mathrm{NA})$ & $91(14)$ & $-0.167(0.136)$ \\
MIROC & $4.6(0.6)$ & $2.6(0.3)$ & $5(0.01)$ & $2.11(0)$ & $84(4)$ & $-0.616(0.051)$ \\
MPI & $5.9(0.9)$ & $2.3(0.2)$ & $5(0)$ & $2.13(0)$ & $85(7)$ & $-0.445(0.083)$ \\
NCAR & $5.5(0.8)$ & $2(0.1)$ & $1.67(0.3)$ & $0.4(0.46)$ & $173(49)$ & $-0.411(0.045)$ \\
\hline
\end{tabular}

\section{DJF SAS ( Equat )}


Table 22: Parameter estimates for the Southern Pacific (ZSP) by season with asymptotic standard errors in parentheses

\begin{tabular}{rrrrrrr}
\hline & $\sigma_{P}$ & $\sigma_{T}$ & $\nu_{P}$ & $\nu_{T}$ & $a^{-1}$ & $\rho$ \\
\hline NCEP/GPCP & $34.2(18.4)$ & $3.4(1.3)$ & $1.58(0.06)$ & $1.22(0.07)$ & $4308(1589)$ & $-0.05(0.048)$ \\
BCC & $5.2(0.7)$ & $1.5(0.2)$ & $2.89(0.2)$ & $3.45(0.27)$ & $430(50)$ & $0.221(0.054)$ \\
GEMS & $8.4(2)$ & $8(3.8)$ & $1.02(0.03)$ & $1.99(0.02)$ & $2637(682)$ & $0.382(0.025)$ \\
GEOS & $13.3(3.5)$ & $2.5(0.7)$ & $1.83(0.07)$ & $1.93(0.06)$ & $1353(246)$ & $0.543(0.03)$ \\
GFDL & $10.3(2.4)$ & $7.6(2.8)$ & $1.94(0.07)$ & $2.78(0.07)$ & $1311(221)$ & $0.11(0.043)$ \\
HAD & $37.7(17.1)$ & $11.9(6.1)$ & $1.65(0.08)$ & $2.12(0.07)$ & $2821(877)$ & $0.571(0.04)$ \\
MIROC & $3(0.2)$ & $0.7(0)$ & $3.95(0.07)$ & $5(4.1)$ & $146(2)$ & $0.395(0.023)$ \\
MPI & $3.5(0.2)$ & $2.6(0.3)$ & $3.07(0.09)$ & $5(45.91)$ & $196(6)$ & $-0.448(0.029)$ \\
NCAR & $174.3(66.5)$ & $11.9(66.5)$ & $1.63(334.43)$ & $1.6(332.25)$ & $8180(10157593804)$ & $-0.002(23.55)$ \\
\hline
\end{tabular}

\section{JJA ZSP ( Mid-S )}

\begin{tabular}{rrrrrrr}
\hline & $\sigma_{P}$ & $\sigma_{T}$ & $\nu_{P}$ & $\nu_{T}$ & $a^{-1}$ & $\rho$ \\
\hline NCEP/GPCP & $17.4(6.6)$ & $5(1.9)$ & $1.36(0.05)$ & $1.4(0.05)$ & $4090(1253)$ & $0.082(0.048)$ \\
BCC & $5.9(0.9)$ & $1.7(0.3)$ & $2.43(0.18)$ & $3.25(0.25)$ & $552(75)$ & $0.093(0.057)$ \\
GEMS & $6.2(1.2)$ & $13.4(7.1)$ & $0.96(0.03)$ & $2.28(0.03)$ & $2322(575)$ & $0.244(0.027)$ \\
GEOS & $22.9(8.9)$ & $3.7(1.1)$ & $1.87(0.06)$ & $1.64(0.07)$ & $1913(446)$ & $0.539(0.03)$ \\
GFDL & $8.3(2)$ & $4.3(1.3)$ & $1.69(0.06)$ & $2.43(0.07)$ & $1420(235)$ & $-0.058(0.044)$ \\
HAD & $33.4(11.1)$ & $9.2(4.9)$ & $1.59(0.07)$ & $1.85(0.06)$ & $3381(1096)$ & $0.005(0.059)$ \\
MIROC & $3.7(0.2)$ & $0.7(0)$ & $3.61(0.06)$ & $5(5.11)$ & $161(2)$ & $0.452(0.021)$ \\
MPI & $4.8(0.3)$ & $2.9(0.3)$ & $3.24(0.1)$ & $5(\mathrm{NA})$ & $199(6)$ & $-0.495(0.028)$ \\
NCAR & $40.2(10.3)$ & $9.7(2.8)$ & $1.4(0.03)$ & $1.68(0.02)$ & $6126(1084)$ & $-0.067(0.021)$ \\
\hline
\end{tabular}

\section{DJF ZSP ( Mid-S )}


Table 23: Parameter estimates for the Equatorial Pacific (ZEP) by season with asymptotic standard errors in parentheses

\begin{tabular}{rrrrrrrr}
\hline & $\sigma_{P}$ & $\sigma_{T}$ & $\nu_{P}$ & $\nu_{T}$ & $a^{-1}$ & $\rho$ \\
\hline NCEP/GPCP & $13.7(2.3)$ & $0.8(0.1)$ & $3.68(0.28)$ & $3.19(0.26)$ & $330(43)$ & $0.063(0.056)$ \\
BCC & $9.8(1.1)$ & $2.1(0.2)$ & $5(0.01)$ & $5(0.03)$ & $244(8)$ & $0.289(0.06)$ \\
GEMS & $9.5(0.7)$ & $1.7(0.2)$ & $2.26(0.07)$ & $3.49(0.11)$ & $298(18)$ & $0.217(0.036)$ \\
GEOS & $8.4(0.9)$ & $0.8(0.1)$ & $4.18(0.31)$ & $2.92(0.14)$ & $219(21)$ & $0.541(0.033)$ \\
GFDL & $10.2(1.1)$ & $1.2(0.1)$ & $4.81(0.19)$ & $4.47(0.14)$ & $229(14)$ & $0.257(0.049)$ \\
HAD & $12.4(1.3)$ & $1(0.1)$ & $2.92(0.67)$ & $1.85(0.51)$ & $225(51)$ & $0.616(0.054)$ \\
MIROC & $11.1(0.7)$ & $0.8(0)$ & $4.98(0.07)$ & $5(0.25)$ & $159(3)$ & $0.199(0.029)$ \\
MPI & $11.2(0.7)$ & $2.1(0.3)$ & $4.21(0.12)$ & $5(18.61)$ & $205(7)$ & $-0.368(0.038)$ \\
NCAR & $11.5(0.9)$ & $0.8(0.1)$ & $3.15(0.05)$ & $3.32(0.06)$ & $266(11)$ & $-0.079(0.024)$ \\
\hline
\end{tabular}

JJA ZEP ( Equat )

\begin{tabular}{rrrrrrrr}
\hline \multicolumn{1}{c}{$\sigma_{P}$} & $\sigma_{T}$ & $\nu_{P}$ & $\nu_{T}$ & $a^{-1}$ & $\rho$ \\
\hline NCEP/GPCP & $15.2(3.1)$ & $0.9(0.1)$ & $2.59(0.21)$ & $2.41(0.22)$ & $514(89)$ & $0.119(0.055)$ \\
BCC & $8.8(0.9)$ & $1.8(0.2)$ & $5(0)$ & $5(0.01)$ & $235(8)$ & $0.528(0.047)$ \\
GEMS & $9.5(0.7)$ & $1.4(0.2)$ & $2.23(0.08)$ & $3.28(0.11)$ & $296(20)$ & $0.479(0.026)$ \\
GEOS & $10.6(1.4)$ & $0.7(0.1)$ & $4.62(0.25)$ & $2.72(0.05)$ & $234(20)$ & $0.454(0.038)$ \\
GFDL & $11.3(1.3)$ & $0.9(0.1)$ & $5(0)$ & $4.14(0)$ & $211(7)$ & $0.449(0.042)$ \\
HAD & $14.1(1.5)$ & $1.1(0.1)$ & $4.76(0.05)$ & $3.35(0)$ & $184(10)$ & $0.439(0.064)$ \\
MIROC & $9.3(0.6)$ & $1(0.1)$ & $4.47(0.08)$ & $5(5.94)$ & $175(3)$ & $0.13(0.03)$ \\
MPI & $13.5(0.9)$ & $1.8(0.2)$ & $4.51(0.14)$ & $5(22.18)$ & $189(6)$ & $-0.057(0.044)$ \\
NCAR & $7.5(0.5)$ & $0.8(0.1)$ & $2.78(0.05)$ & $3.34(0.07)$ & $263(12)$ & $-0.11(0.024)$ \\
\hline
\end{tabular}

DJF ZEP ( Equat ) 
Table 24: Parameter estimates for the Northern Pacific (ZNP) by season with asymptotic standard errors in parentheses

\begin{tabular}{rrrrrrr}
\hline \multicolumn{1}{c}{$\sigma_{P}$} & $\sigma_{T}$ & $\nu_{P}$ & $\nu_{T}$ & $a^{-1}$ & $\rho$ \\
\hline NCEP/GPCP & $17.4(5.8)$ & $24.3(15.6)$ & $0.9(0.05)$ & $1.78(0.05)$ & $4954(1931)$ & $0.03(0.054)$ \\
BCC & $5.5(1.1)$ & $2.1(0.5)$ & $2.22(0.17)$ & $2.89(0.21)$ & $627(111)$ & $0.012(0.061)$ \\
GEMS & $7.2(1.5)$ & $5.1(1.7)$ & $0.93(0.04)$ & $1.76(0.03)$ & $1799(414)$ & $0.411(0.026)$ \\
GEOS & $32(9.6)$ & $14.8(6.5)$ & $1.28(0.06)$ & $1.82(0.05)$ & $2732(746)$ & $-0.851(0.016)$ \\
GFDL & $25(7.4)$ & $5.9(2)$ & $1.83(0.07)$ & $1.99(0.07)$ & $1911(402)$ & $-0.067(0.051)$ \\
HAD & $31.8(14.3)$ & $39.9(20.3)$ & $1.62(0.09)$ & $2.1(0.1)$ & $2761(853)$ & $-0.088(0.064)$ \\
MIROC & $4(0.2)$ & $1.4(0.1)$ & $3.38(0.07)$ & $5(17.37)$ & $173(3)$ & $0.121(0.029)$ \\
MPI & $5.5(0.3)$ & $1.8(0.2)$ & $3.64(0.11)$ & $5(31.83)$ & $194(7)$ & $-0.348(0.035)$ \\
NCAR & $28.3(10.8)$ & $4.1(1.5)$ & $1.47(0.03)$ & $1.49(0.03)$ & $2803(785)$ & $-0.282(0.021)$ \\
\hline
\end{tabular}

\section{JJA ZNP ( Mid-N )}

\begin{tabular}{rrrrrrrr}
\hline & $\sigma_{P}$ & $\sigma_{T}$ & $\nu_{P}$ & $\nu_{T}$ & $a^{-1}$ & $\rho$ \\
\hline NCEP/GPCP & $12.6(4.6)$ & $28.3(18.7)$ & $0.76(0.04)$ & $1.58(0.03)$ & $8947(4122)$ & $-0.018(0.052)$ \\
BCC & $7(1.1)$ & $2(0.4)$ & $2.7(0.16)$ & $3.13(0.19)$ & $537(67)$ & $0.225(0.059)$ \\
GEMS & $29(10.3)$ & $29.4(18.2)$ & $1.11(0.04)$ & $1.83(0.03)$ & $4172(1487)$ & $0.546(0.022)$ \\
GEOS & $51.1(19.6)$ & $25.5(12.1)$ & $1.41(0.06)$ & $1.73(0.05)$ & $3953(1178)$ & $-0.772(0.025)$ \\
GFDL & $73.1(29.8)$ & $28.1(13.8)$ & $2.01(0.06)$ & $2.34(0.07)$ & $2846(697)$ & $0.063(0.049)$ \\
HAD & $54(21.6)$ & $29.9(13.2)$ & $1.94(0.08)$ & $2.35(0.1)$ & $2457(602)$ & $0.583(0.043)$ \\
MIROC & $4.2(0.3)$ & $1.5(0.1)$ & $2.89(0.07)$ & $3.95(0.13)$ & $268(12)$ & $0.315(0.027)$ \\
MPI & $4.8(0.4)$ & $1.1(0.1)$ & $3.82(0.16)$ & $4.37(0.29)$ & $192(11)$ & $-0.22(0.041)$ \\
NCAR & $112.6(62.1)$ & $7.7(62.1)$ & $1.57(292.6)$ & $1.32(259.45)$ & $6722(5708297223)$ & $-0.154(22.085)$ \\
\hline
\end{tabular}

\section{DJF ZNP ( Mid-N )}


Table 25: Parameter estimates for the East Equatorial Atlantic (ZEA) by season with asymptotic standard errors in parentheses

\begin{tabular}{rrrrrrrr}
\hline & $\sigma_{P}$ & $\sigma_{T}$ & $\nu_{P}$ & $\nu_{T}$ & $a^{-1}$ & $\rho$ \\
\hline NCEP/GPCP & $4.8(1.1)$ & $1.4(0.5)$ & $1.18(0.17)$ & $1.91(0.18)$ & $731(248)$ & $-0.338(0.076)$ \\
BCC & $7.1(2)$ & $2.2(0.7)$ & $2.85(0.45)$ & $3.56(0.8)$ & $441(125)$ & $0.061(0.101)$ \\
GEMS & $12.4(2.8)$ & $2.9(1)$ & $1.66(0.07)$ & $2.3(0.07)$ & $793(148)$ & $0.363(0.044)$ \\
GEOS & $15.7(4.6)$ & $1.4(0.3)$ & $2.3(0.2)$ & $1.71(0.18)$ & $615(146)$ & $0.105(0.073)$ \\
GFDL & $7.5(1.9)$ & $2.5(0.7)$ & $2.43(0.2)$ & $2.76(0.21)$ & $596(115)$ & $-0.39(0.064)$ \\
HAD & $11.3(3.6)$ & $3.1(1.4)$ & $1.21(0.12)$ & $1.76(0.1)$ & $1733(617)$ & $0.164(0.096)$ \\
MIROC & $6.8(0.8)$ & $0.9(0.1)$ & $4.98(0.17)$ & $5(0.45)$ & $129(5)$ & $0.039(0.05)$ \\
MPI & $7.3(0.7)$ & $2(0.4)$ & $4.15(0.2)$ & $5(14.95)$ & $190(10)$ & $-0.376(0.053)$ \\
NCAR & $5.2(0.9)$ & $5.5(1.7)$ & $1.34(0.05)$ & $2.1(0.05)$ & $790(139)$ & $0.067(0.036)$ \\
\hline
\end{tabular}

JJA ZEA ( Equat )

\begin{tabular}{rrrrrrrr}
\hline & $\sigma_{P}$ & $\sigma_{T}$ & $\nu_{P}$ & $\nu_{T}$ & $a^{-1}$ & $\rho$ \\
\hline NCEP/GPCP & $14.3(4.5)$ & $2.3(0.9)$ & $1.64(0.16)$ & $1.92(0.15)$ & $1081(329)$ & $-0.261(0.079)$ \\
BCC & $10.6(4.2)$ & $1.8(0.6)$ & $2.45(0.36)$ & $2.44(0.37)$ & $658(228)$ & $0.122(0.093)$ \\
GEMS & $6.7(1.2)$ & $2.7(0.9)$ & $1.44(0.08)$ & $2.55(0.07)$ & $665(117)$ & $0.409(0.042)$ \\
GEOS & $42.7(20.5)$ & $1.4(0.4)$ & $2.03(0.15)$ & $1.28(0.13)$ & $1155(359)$ & $0.151(0.069)$ \\
GFDL & $11.8(2.9)$ & $1(0.2)$ & $3.6(0.34)$ & $3.31(0.27)$ & $307(49)$ & $0.362(0.066)$ \\
HAD & $13.5(4.1)$ & $1.1(0.2)$ & $1.56(0.19)$ & $1.48(0.17)$ & $969(310)$ & $0.346(0.084)$ \\
MIROC & $6.9(1)$ & $1.6(0.2)$ & $3.78(0.23)$ & $4.49(0.63)$ & $183(18)$ & $0.347(0.043)$ \\
MPI & $9.5(0.9)$ & $2.7(0.5)$ & $4.09(0.19)$ & $5(14.99)$ & $207(10)$ & $-0.257(0.06)$ \\
NCAR & $23.1(6.9)$ & $4.4(1.4)$ & $1.92(0.05)$ & $1.92(0.05)$ & $930(178)$ & $-0.186(0.035)$ \\
\hline
\end{tabular}

DJF ZEA ( Equat ) 
Table 26: Parameter estimates for the West Equatorial Atlantic (ZAE) by season with asymptotic standard errors in parentheses

\begin{tabular}{rrrrrrrr}
\hline & $\sigma_{P}$ & $\sigma_{T}$ & $\nu_{P}$ & $\nu_{T}$ & $a^{-1}$ & $\rho$ \\
\hline NCEP/GPCP & $19.8(7.2)$ & $1(0.3)$ & $2.36(0.38)$ & $2.35(0.4)$ & $496(161)$ & $-0.02(0.108)$ \\
BCC & $9.3(1.7)$ & $1(0.2)$ & $4.77(0.35)$ & $5(9.14)$ & $249(15)$ & $0.42(0.1)$ \\
GEMS & $8(1.4)$ & $1.1(0.3)$ & $1.57(0.1)$ & $2.74(0.13)$ & $457(69)$ & $0.3(0.057)$ \\
GEOS & $7.4(1.1)$ & $1(0.2)$ & $4.36(0.52)$ & $4.44(0.8)$ & $194(28)$ & $0.159(0.091)$ \\
GFDL & $18.1(5.4)$ & $2.5(0.9)$ & $2.32(0.2)$ & $2.64(0.2)$ & $769(185)$ & $0.348(0.082)$ \\
HAD & $18.4(7.7)$ & $1.4(0.5)$ & $2.07(0.25)$ & $2.37(0.27)$ & $723(225)$ & $0.583(0.082)$ \\
MIROC & $11.8(1.4)$ & $0.9(0.1)$ & $4.77(0.19)$ & $5(6.04)$ & $152(5)$ & $0.291(0.054)$ \\
MPI & $12.2(1.4)$ & $2.1(0.4)$ & $4.45(0.25)$ & $5(24.58)$ & $186(11)$ & $0.06(0.077)$ \\
NCAR & $26(6.8)$ & $1.6(0.3)$ & $2.37(0.06)$ & $2.22(0.07)$ & $633(85)$ & $-0.24(0.042)$ \\
\hline
\end{tabular}

JJA ZAE ( Equat )

\begin{tabular}{rrrrrrrr}
\hline & $\sigma_{P}$ & $\sigma_{T}$ & $\nu_{P}$ & $\nu_{T}$ & $a^{-1}$ & $\rho$ \\
\hline NCEP/GPCP & $64.4(44)$ & $1.4(0.5)$ & $2.95(0.32)$ & $2.24(0.3)$ & $704(254)$ & $-0.15(0.103)$ \\
BCC & $6.1(1.2)$ & $1.1(0.2)$ & $4.06(0.37)$ & $5(25.53)$ & $247(17)$ & $0.53(0.089)$ \\
GEMS & $14.9(2.8)$ & $1.5(0.4)$ & $1.8(0.11)$ & $2.61(0.12)$ & $549(96)$ & $0.385(0.054)$ \\
GEOS & $4.8(0.8)$ & $0.8(0.1)$ & $2.47(0.46)$ & $3.16(0.77)$ & $281(74)$ & $-0.217(0.082)$ \\
GFDL & $34(13.9)$ & $2.3(0.8)$ & $2.92(0.32)$ & $2.56(0.26)$ & $614(191)$ & $0.635(0.058)$ \\
HAD & $22.8(7.6)$ & $1.4(0.4)$ & $2.3(0.23)$ & $2.23(0.25)$ & $697(191)$ & $0.481(0.092)$ \\
MIROC & $12.8(1.7)$ & $1(0.2)$ & $4.83(0.21)$ & $5(4.45)$ & $159(8)$ & $0.195(0.057)$ \\
MPI & $5.4(0.6)$ & $1.3(0.2)$ & $3.67(0.21)$ & $5(31.26)$ & $178(10)$ & $0.02(0.078)$ \\
NCAR & $17.8(4.1)$ & $1.2(0.2)$ & $2.6(0.07)$ & $2.69(0.08)$ & $415(46)$ & $-0.09(0.044)$ \\
\hline
\end{tabular}

DJF ZAE ( Equat ) 
Table 27: Parameter estimates for the North Atlantic (ZAN) by season with asymptotic standard errors in parentheses

\begin{tabular}{rrrrrrrr}
\hline & $\sigma_{P}$ & $\sigma_{T}$ & $\nu_{P}$ & $\nu_{T}$ & $a^{-1}$ & $\rho$ \\
\hline NCEP/GPCP & $9.5(5.5)$ & $2.2(1.3)$ & $1.27(0.1)$ & $1.34(0.12)$ & $2733(1488)$ & $-0.109(0.09)$ \\
BCC & $2.7(0.5)$ & $2.1(0.6)$ & $2.74(0.36)$ & $4.47(1.64)$ & $373(75)$ & $-0.151(0.105)$ \\
GEMS & $4.9(1.2)$ & $18.4(8.9)$ & $1.16(0.07)$ & $2.78(0.08)$ & $1055(243)$ & $0.518(0.042)$ \\
GEOS & $10.7(4.4)$ & $1.5(0.6)$ & $1.95(0.12)$ & $1.75(0.13)$ & $1166(349)$ & $-0.181(0.073)$ \\
GFDL & $24.2(10.4)$ & $9.5(4.6)$ & $2.14(0.1)$ & $2.64(0.13)$ & $1555(384)$ & $0.38(0.074)$ \\
HAD & $14.4(8.2)$ & $10.1(5.3)$ & $1.31(0.12)$ & $1.71(0.1)$ & $3027(1253)$ & $0.445(0.083)$ \\
MIROC & $3.3(0.3)$ & $0.9(0.1)$ & $3.81(0.13)$ & $5(16.41)$ & $150(5)$ & $0.406(0.041)$ \\
MPI & $2.6(0.3)$ & $1.2(0.2)$ & $3.15(0.19)$ & $5(14.78)$ & $164(9)$ & $-0.347(0.06)$ \\
NCAR & $5.3(1.3)$ & $7.1(3.2)$ & $1.56(0.05)$ & $2.73(0.05)$ & $1099(218)$ & $-0.167(0.037)$ \\
\hline
\end{tabular}

\section{JJA ZAN ( Mid-N )}

\begin{tabular}{rrrrrrrr}
\hline \multicolumn{1}{c}{$\sigma_{P}$} & $\sigma_{T}$ & $\nu_{P}$ & $\nu_{T}$ & $a^{-1}$ & $\rho$ \\
\hline NCEP/GPCP & $3.7(1.6)$ & $6.6(4)$ & $0.94(0.1)$ & $1.75(0.09)$ & $2564(1263)$ & $-0.025(0.092)$ \\
BCC & $1.8(0.4)$ & $1.3(0.3)$ & $2.69(0.47)$ & $4.4(2.94)$ & $295(73)$ & $0.246(0.101)$ \\
GEMS & $5.1(1.3)$ & $14.5(6.9)$ & $1.11(0.06)$ & $2.59(0.06)$ & $1317(319)$ & $0.4(0.046)$ \\
GEOS & $5.5(1.6)$ & $3.4(1.1)$ & $2.15(0.17)$ & $2.61(0.18)$ & $734(162)$ & $-0.118(0.082)$ \\
GFDL & $4.4(1.3)$ & $4.8(1.7)$ & $2.26(0.19)$ & $3.58(0.34)$ & $609(133)$ & $0.462(0.072)$ \\
HAD & $22.1(9.6)$ & $13.4(8.4)$ & $1.48(0.14)$ & $1.95(0.11)$ & $2641(1041)$ & $0.868(0.024)$ \\
MIROC & $2.5(0.2)$ & $1.3(0.2)$ & $3.19(0.1)$ & $5(\mathrm{NA})$ & $184(6)$ & $0.454(0.039)$ \\
MPI & $2.6(0.3)$ & $0.9(0.1)$ & $3.36(0.2)$ & $5(15.51)$ & $153(7)$ & $0.191(0.066)$ \\
NCAR & $3.2(0.7)$ & $4.2(1.3)$ & $1.43(0.05)$ & $2.67(0.06)$ & $958(158)$ & $-0.001(0.038)$ \\
\hline
\end{tabular}

\section{DJF ZAN ( Mid-N )}


Table 28: Parameter estimates for the Far North Atlantic (ZAN) by season with asymptotic standard errors in parentheses

\begin{tabular}{rrrrrrrr}
\hline & $\sigma_{P}$ & $\sigma_{T}$ & $\nu_{P}$ & $\nu_{T}$ & $a^{-1}$ & $\rho$ \\
\hline NCEP/GPCP & $2.5(0.3)$ & $1.9(0.4)$ & $2.06(0.21)$ & $5(21.97)$ & $194(12)$ & $0.131(0.097)$ \\
BCC & $2.4(0.5)$ & $2.1(0.4)$ & $3.17(0.34)$ & $3.01(0.34)$ & $303(52)$ & $0.118(0.095)$ \\
GEMS & $2.2(0.3)$ & $2.9(0.6)$ & $1.36(0.09)$ & $2.21(0.08)$ & $390(59)$ & $0.227(0.051)$ \\
GEOS & $3.1(0.6)$ & $2(0.4)$ & $1.1(0.14)$ & $1.38(0.13)$ & $525(144)$ & $0.219(0.07)$ \\
GFDL & $6.4(2.2)$ & $3.1(1)$ & $1.79(0.11)$ & $1.73(0.1)$ & $936(233)$ & $-0.36(0.072)$ \\
HAD & $4(1.2)$ & $3(0.9)$ & $1.86(0.27)$ & $2.17(0.31)$ & $548(168)$ & $0.083(0.101)$ \\
MIROC & $3.2(0.6)$ & $1.7(0.2)$ & $2.74(0.11)$ & $2.7(0.1)$ & $248(22)$ & $0.053(0.051)$ \\
MPI & $6.7(1.9)$ & $2.4(0.5)$ & $2.05(0.14)$ & $1.96(0.12)$ & $494(93)$ & $-0.278(0.067)$ \\
NCAR & $9(2.7)$ & $2.7(0.7)$ & $1.72(0.06)$ & $1.68(0.04)$ & $927(167)$ & $0(0.037)$ \\
\hline
\end{tabular}

JJA ZNA ( Mid-N )

\begin{tabular}{rrrrrrr}
\hline & $\sigma_{P}$ & $\sigma_{T}$ & $\nu_{P}$ & $\nu_{T}$ & $a^{-1}$ & $\rho$ \\
\hline NCEP/GPCP & $2.1(0.2)$ & $3.5(0.8)$ & $1.4(0.17)$ & $5(21.34)$ & $211(13)$ & $0.124(0.099)$ \\
BCC & $5.5(1.5)$ & $4.4(1.1)$ & $2.44(0.21)$ & $2(0.17)$ & $577(124)$ & $0.476(0.074)$ \\
GEMS & $7.9(3)$ & $3.9(1.4)$ & $1.31(0.08)$ & $1.45(0.07)$ & $1157(374)$ & $0.368(0.046)$ \\
GEOS & $5.2(1.9)$ & $3.1(0.9)$ & $1.06(0.1)$ & $0.82(0.1)$ & $1566(644)$ & $-0.358(0.072)$ \\
GFDL & $3.9(1.2)$ & $3(0.8)$ & $1.94(0.16)$ & $1.99(0.16)$ & $540(132)$ & $0.582(0.053)$ \\
HAD & $3.9(1.1)$ & $3.7(1.5)$ & $1.31(0.17)$ & $1.89(0.15)$ & $887(296)$ & $0.508(0.073)$ \\
MIROC & $4.4(0.8)$ & $2.4(0.4)$ & $2.89(0.1)$ & $3.03(0.1)$ & $240(21)$ & $0.289(0.047)$ \\
MPI & $5.3(1.5)$ & $2.5(0.5)$ & $1.89(0.14)$ & $2.02(0.13)$ & $398(77)$ & $0.358(0.063)$ \\
NCAR & $4.5(0.9)$ & $5.3(1.3)$ & $1.79(0.05)$ & $2.24(0.05)$ & $530(74)$ & $0.599(0.024)$ \\
\hline
\end{tabular}

\section{DJF ZNA ( Mid-N )}


Table 29: Parameter estimates for the Southern Atlantic (ZSA) by season with asymptotic standard errors in parentheses

\begin{tabular}{rrrrrrr}
\hline & $\sigma_{P}$ & $\sigma_{T}$ & $\nu_{P}$ & $\nu_{T}$ & $a^{-1}$ & $\rho$ \\
\hline NCEP/GPCP & $11.1(5.1)$ & $6.4(3.1)$ & $1.25(0.06)$ & $1.53(0.09)$ & $3170(1309)$ & $-0.006(0.065)$ \\
BCC & $3.9(0.6)$ & $3.2(0.7)$ & $2.64(0.22)$ & $3.03(0.24)$ & $435(64)$ & $0.531(0.051)$ \\
GEMS & $3.4(0.5)$ & $2.6(0.7)$ & $1.23(0.07)$ & $2.1(0.06)$ & $709(125)$ & $0.504(0.029)$ \\
GEOS & $11.1(4.2)$ & $7.4(3.6)$ & $2.53(0.16)$ & $2.73(0.19)$ & $727(177)$ & $0.201(0.062)$ \\
GFDL & $8.9(2.7)$ & $4(1.4)$ & $2.13(0.11)$ & $2.28(0.12)$ & $1056(230)$ & $0.568(0.04)$ \\
HAD & $5.3(1.2)$ & $2.7(1.1)$ & $1.29(0.14)$ & $1.86(0.13)$ & $1108(394)$ & $0.663(0.042)$ \\
MIROC & $3.4(0.3)$ & $1.1(0.1)$ & $3.38(0.11)$ & $4.24(0.17)$ & $192(9)$ & $0.474(0.029)$ \\
MPI & $3.7(0.3)$ & $1.6(0.2)$ & $3.87(0.13)$ & $5(8.11)$ & $164(5)$ & $0.098(0.048)$ \\
NCAR & $16(2.9)$ & $25(6.1)$ & $1.93(0.04)$ & $2.64(0.04)$ & $1219(124)$ & $0.12(0.028)$ \\
\hline
\end{tabular}

JJA ZSA ( Mid-S )

\begin{tabular}{rrrrrrrr}
\hline \multicolumn{1}{c}{$\sigma_{P}$} & $\sigma_{T}$ & $\nu_{P}$ & $\nu_{T}$ & $a^{-1}$ & $\rho$ \\
\hline NCEP/GPCP & $12.8(5.4)$ & $9(4.1)$ & $1.32(0.07)$ & $1.76(0.1)$ & $2483(910)$ & $0.056(0.065)$ \\
BCC & $5.5(0.9)$ & $3.1(0.6)$ & $2.8(0.19)$ & $3.27(0.24)$ & $457(57)$ & $0.021(0.072)$ \\
GEMS & $4.1(0.8)$ & $8.2(3.5)$ & $1.08(0.05)$ & $2.05(0.04)$ & $1405(326)$ & $0.318(0.034)$ \\
GEOS & $20.1(6.5)$ & $3.5(0.9)$ & $2.94(0.12)$ & $2.54(0.14)$ & $686(110)$ & $-0.074(0.056)$ \\
GFDL & $7(1.7)$ & $2.8(0.7)$ & $2.16(0.1)$ & $2.38(0.12)$ & $824(131)$ & $0.227(0.055)$ \\
HAD & $10.3(3.1)$ & $3.8(1.4)$ & $1.51(0.11)$ & $1.57(0.11)$ & $1763(606)$ & $0.183(0.075)$ \\
MIROC & $3.2(0.2)$ & $1.3(0.1)$ & $3.22(0.1)$ & $4.41(0.23)$ & $199(9)$ & $0.289(0.033)$ \\
MPI & $3.9(0.3)$ & $2(0.2)$ & $3.68(0.12)$ & $5(8.42)$ & $177(6)$ & $-0.272(0.046)$ \\
NCAR & $6.7(1.2)$ & $3.9(1)$ & $1.85(0.06)$ & $2.54(0.05)$ & $648(78)$ & $0.135(0.028)$ \\
\hline
\end{tabular}

DJF ZSA ( Mid-S ) 
Table 30: Parameter estimates for the Equatorial Indian Ocean (ZEI) by season with asymptotic standard errors in parentheses

\begin{tabular}{rrrrrrr}
\hline & $\sigma_{P}$ & $\sigma_{T}$ & $\nu_{P}$ & $\nu_{T}$ & $a^{-1}$ & $\rho$ \\
\hline NCEP/GPCP & $6.3(0.9)$ & $1.9(0.5)$ & $1.41(0.17)$ & $2.51(0.23)$ & $511(113)$ & $-0.179(0.064)$ \\
BCC & $11.9(3.3)$ & $0.9(0.1)$ & $3.14(0.32)$ & $2.05(0.25)$ & $447(86)$ & $-0.015(0.073)$ \\
GEMS & $9.1(1.5)$ & $0.9(0.1)$ & $1.3(0.09)$ & $1.07(0.09)$ & $531(103)$ & $0.168(0.038)$ \\
GEOS & $27(9)$ & $1.6(0.3)$ & $1.55(0.09)$ & $0.78(0.13)$ & $1243(349)$ & $-0.556(0.038)$ \\
GFDL & $27.6(12.7)$ & $2.5(0.8)$ & $1.51(0.08)$ & $1.03(0.12)$ & $2025(738)$ & $-0.061(0.058)$ \\
HAD & $16.7(5.8)$ & $1.3(0.4)$ & $1.28(0.16)$ & $1.13(0.18)$ & $1196(514)$ & $-0.018(0.081)$ \\
MIROC & $7.6(0.6)$ & $1.2(0.1)$ & $2.53(0.13)$ & $2.85(0.16)$ & $189(14)$ & $-0.231(0.035)$ \\
MPI & $7.5(0.7)$ & $1.4(0.2)$ & $1.5(0.17)$ & $2.33(0.18)$ & $311(54)$ & $0.037(0.049)$ \\
NCAR & $7.9(1.4)$ & $1.8(0.3)$ & $1.34(0.04)$ & $1.17(0.05)$ & $791(129)$ & $-0.125(0.027)$ \\
\hline
\end{tabular}

JJA ZEI ( Equat )

\begin{tabular}{rrrrrrrr}
\hline \multicolumn{1}{c}{$\sigma_{P}$} & $\sigma_{T}$ & $\nu_{P}$ & $\nu_{T}$ & $a^{-1}$ & $\rho$ \\
\hline NCEP/GPCP & $12.1(3.7)$ & $5.1(2.5)$ & $1.11(0.09)$ & $1.8(0.11)$ & $1660(648)$ & $-0.163(0.065)$ \\
BCC & $15.8(4.7)$ & $1.3(0.3)$ & $2.09(0.18)$ & $1.62(0.17)$ & $811(205)$ & $0.35(0.066)$ \\
GEMS & $24.5(11.4)$ & $3.1(1.4)$ & $0.9(0.04)$ & $0.85(0.04)$ & $4357(2461)$ & $0.553(0.027)$ \\
GEOS & $139.6(101.5)$ & $3.8(1.5)$ & $1.31(0.06)$ & $0.66(0.12)$ & $6931(4052)$ & $-0.47(0.046)$ \\
GFDL & $33.3(15.9)$ & $3(1.2)$ & $1.53(0.08)$ & $1.15(0.09)$ & $2519(1025)$ & $0.289(0.054)$ \\
HAD & $33.3(17.6)$ & $2.7(1.1)$ & $1.21(0.09)$ & $0.99(0.1)$ & $3224(1573)$ & $0.336(0.072)$ \\
MIROC & $9.8(1.3)$ & $1.1(0.1)$ & $2.23(0.09)$ & $1.91(0.09)$ & $349(34)$ & $-0.241(0.035)$ \\
MPI & $6.8(0.4)$ & $1.1(0.2)$ & $3.24(0.49)$ & $4.62(1.86)$ & $144(20)$ & $0.438(0.046)$ \\
NCAR & $22.3(8)$ & $7.6(2.8)$ & $1.4(0.04)$ & $1.39(0.04)$ & $1616(462)$ & $-0.171(0.027)$ \\
\hline
\end{tabular}

DJF ZEI ( Equat ) 
Table 31: Parameter estimates for the Southern Indian Ocean (ZSI) by season with asymptotic standard errors in parentheses

\begin{tabular}{rrrrrrr}
\hline & $\sigma_{P}$ & $\sigma_{T}$ & $\nu_{P}$ & $\nu_{T}$ & $a^{-1}$ & $\rho$ \\
\hline NCEP/GPCP & $1.3(0.1)$ & $1.1(0.2)$ & $2.07(0.12)$ & $5(27.31)$ & $220(9)$ & $0.038(0.06)$ \\
BCC & $3.4(0.5)$ & $2(0.3)$ & $3.27(0.23)$ & $3.64(0.29)$ & $311(33)$ & $0.429(0.054)$ \\
GEMS & $16.8(8.2)$ & $5.2(3)$ & $1.06(0.04)$ & $1.21(0.03)$ & $4313(2135)$ & $0.448(0.028)$ \\
GEOS & $3(0.6)$ & $1.9(0.5)$ & $1.29(0.09)$ & $1.71(0.08)$ & $803(188)$ & $-0.27(0.059)$ \\
GFDL & $5(0.9)$ & $1.6(0.3)$ & $2.19(0.11)$ & $2.38(0.1)$ & $561(71)$ & $0.529(0.038)$ \\
HAD & $13(4.8)$ & $6.2(3)$ & $1.24(0.1)$ & $1.42(0.1)$ & $2390(1236)$ & $0.683(0.036)$ \\
MIROC & $4.4(0.4)$ & $0.6(0)$ & $4.02(0.11)$ & $4.11(0.11)$ & $162(7)$ & $0.399(0.028)$ \\
MPI & $3.2(0.4)$ & $0.8(0.1)$ & $1.82(0.1)$ & $1.79(0.09)$ & $354(38)$ & $0.325(0.044)$ \\
NCAR & $7.1(1.8)$ & $2.5(0.7)$ & $1.76(0.04)$ & $1.89(0.03)$ & $976(163)$ & $0.283(0.023)$ \\
\hline
\end{tabular}

JJA ZSI ( Mid-S )

\begin{tabular}{rrrrrrr}
\hline & $\sigma_{P}$ & $\sigma_{T}$ & $\nu_{P}$ & $\nu_{T}$ & $a^{-1}$ & $\rho$ \\
\hline NCEP/GPCP & $2.5(0.4)$ & $2.7(0.7)$ & $1.36(0.12)$ & $2.86(0.18)$ & $615(123)$ & $0.008(0.06)$ \\
BCC & $6.7(1.2)$ & $3(0.6)$ & $2.52(0.14)$ & $2.77(0.13)$ & $586(72)$ & $0.195(0.063)$ \\
GEMS & $5(1.1)$ & $3.5(1.3)$ & $1.1(0.05)$ & $1.76(0.04)$ & $1312(334)$ & $0.186(0.034)$ \\
GEOS & $11.7(4.3)$ & $14.7(8.6)$ & $1.05(0.05)$ & $1.62(0.05)$ & $3707(1462)$ & $0.312(0.046)$ \\
GFDL & $9.4(2.6)$ & $3(0.9)$ & $1.76(0.09)$ & $1.86(0.08)$ & $1204(267)$ & $-0.127(0.055)$ \\
HAD & $15.1(6.9)$ & $7.3(3)$ & $1.35(0.09)$ & $1.41(0.09)$ & $3032(1345)$ & $0.442(0.056)$ \\
MIROC & $5.6(0.8)$ & $1.1(0.1)$ & $2.61(0.09)$ & $2.69(0.07)$ & $351(25)$ & $0.149(0.032)$ \\
MPI & $6.2(0.8)$ & $1.8(0.3)$ & $2.08(0.1)$ & $2.27(0.08)$ & $458(47)$ & $-0.588(0.03)$ \\
NCAR & $26.2(10.5)$ & $4(1.4)$ & $1.67(0.04)$ & $1.61(0.03)$ & $1964(499)$ & $0.072(0.025)$ \\
\hline
\end{tabular}

DJF ZSI ( Mid-S ) 